Research Article

\title{
The Subchronic Toxic Effects of Mosla chinensis Maxim in Normal Rats
}

\author{
Dan Lei, ${ }^{1}$ Longxue Li, ${ }^{1}$ Shenghong Huang, ${ }^{1}$ Li Liu $\mathbb{D}^{2},{ }^{2}$ Pingdong Cai, ${ }^{3}$ Zhouyang Gu, \\ Kun Shu, ${ }^{1}$ Shouming Li $\odot$, ${ }^{4}$ Tao Hong ${ }^{1},{ }^{1}$ and Zhiyong Liu $\oplus^{1,5}$ \\ ${ }^{1}$ Jiangxi University of Chinese Medicine, Nanchang 330004, China \\ ${ }_{2}^{2}$ Jiangxi Medical Service Guidance Center, Nanchang 330006, China \\ ${ }^{3}$ School of Science and Technology, Jiangxi University of Traditional Chinese Medicine, Nanchang 330004, China \\ ${ }^{4} J i a n g x i$ Children's Hospital, Nanchang 330006, China \\ ${ }_{5}^{5}$ Jiangxi Provincial Key Laboratory of Traditional Chinese Medicine Pharmacology, Nanchang 330004, China
}

Correspondence should be addressed to Li Liu; 13870803370@163.com, Shouming Li; lishouming0791@163.com, Tao Hong; 372729181@qq.com, and Zhiyong Liu; liuzhiyong0791@163.com

Received 24 August 2020; Revised 21 November 2020; Accepted 8 December 2020; Published 28 December 2020

Academic Editor: Elena Baralla

Copyright (C) 2020 Dan Lei et al. This is an open access article distributed under the Creative Commons Attribution License, which permits unrestricted use, distribution, and reproduction in any medium, provided the original work is properly cited.

\begin{abstract}
Background. The aim of this work was to study the toxic effects and target organs of Mosla chinensis Maxim (MCM) in rats and provide theoretical basis for clinical medication. Methods. The subchronic toxicity study was conducted on 60 male and female SD rats using the fixed-dose method for the treatment groups and 20 male and female SD rats for the control. At the subchronic toxicity study, the water extract of MCM with fixed doses of $0.2 \mathrm{~g} / \mathrm{kg} / \mathrm{day}, 2 \mathrm{~g} / \mathrm{kg} / \mathrm{day}$, and $20 \mathrm{~g} / \mathrm{kg} / \mathrm{day}$ was administered for 90 days intragastric, and the control group was given the same amount of distilled water. After 90 days, the general conditions of the rats were observed. Assessment on safety of the extract was conducted by a subchronic toxicity test which mainly examined alteration occurrence in gut flora and urine metabolism. Results. There was no significant difference in physical signs, reactivity, and stool characteristics in the four groups. Compared with the control group, the number of red blood cells in the male $2 \mathrm{~g} / \mathrm{kg} /$ day group and the female $0.2 \mathrm{~g} / \mathrm{kg} /$ day group was significantly different $(P<0.05)$. The detection of serum biochemical indicators showed that MCM has an effect on liver and kidney function but has no physiological significance. The level of lowdensity lipoprotein in male rats was lower than that in the control group $(P<0.05)$. Compared with the control group, the blood glucose levels of female rats in the $0.2 \mathrm{~g} / \mathrm{kg} / \mathrm{day}, 2 \mathrm{~g} / \mathrm{kg} /$ day, and $20 \mathrm{~g} / \mathrm{kg} /$ day groups were significantly increased $(P<0.05)$. As far as the diversity of intestinal flora is concerned, feeding MCM for 90 days has an influence on the distribution of intestinal flora. The content of lactic acid bacteria increased, and the ratio of hard bacteria to Bacteroides (f/b) was also affected, but there was no significant difference. Conclusions. These findings showed that the long-term intragastric administration of the MCM is safe to use within its dose recommendation. But it could have a slight effect on the metabolism of uric acid by changing the composition of intestinal flora and affecting the metabolism of tryptophan.
\end{abstract}

\section{Background}

Mosla chinensis Maxim cv. Jiangxiangru (MCM) is mainly distributed in Jiangxi province. Also, as the unique resource in Jiangxi, it belongs to the member of the Labiatae family and is usually used for both food and medicine. The whole herb can be used as medicine and is clinically used to treat acute gastroenteritis, abdominal pain, and diarrhea. The main chemical components of Elsholtzia are volatile oil, followed by flavonoids and glycosides, triterpenes and steroids, and a small amount of coumarins, lignans, cyanogenic glycoside, organic acids, and aliphatic hydrocarbons. Its volatile oil has many pharmacological effects, such as pesticides, antioxidants, and antibacterial [1]. It is one of the components of Changyanning, a medicine for gastroenteritis [2]. Due to its significant anti-inflammatory and other active effects, MCM has been listed as a traditional Chinese medicine with both medicinal and food functions. However, 
when some Chinese herbal medicines are transformed and metabolized by the liver, themselves or their metabolites can directly or indirectly cause liver damage or cause toxic damage to hepatocytes through immune-mediated mechanisms [3]. For example, Chinese herbal medicines such as Polygonum multiflorum, Celandine, Xiaochaihu decoction, and Huangyaozi have potential liver toxicity [4]. For scientific evaluation of the safety of MCM and mastering its security, in this study, the water extract of MCM was used. Through 90 days of gastric administration, the changes of intestinal flora were observed by using 16S DNA sequencing technology, and the changes of urine metabolism were observed by UHPLC-MS, in order to provide the basis for comprehensively understanding the safety evaluation of MCM.

\section{Materials and Methods}

2.1. Experimental Animals. The SD rats originated from the National Laboratory Animal Seed Center and bred at the Experimental Animal Science and Technology Center of Jiangxi University of Traditional Chinese Medicine, of which half were male and half were female, 8 weeks old, and weighed $100 \pm 10 \mathrm{~g}$. Experimental rats were raised in a SPF barrier system. In this experiment, a barrier housing facility was used in accordance with the national standard laboratory animal requirements of environment and housing facilities. The rats were housed in acrylic cages lined with wood shavings at constant room temperature $\left(23 \pm 1^{\circ} \mathrm{C}\right)$ and maintained on a $12 \mathrm{~h}$ light and $12 \mathrm{~h}$ dark cycle. The care of the laboratory animals and the animal experimental operation were performed in accordance with the committee of the Jiangxi University of Traditional Chinese Medicine.

2.2. MCM Water Extract Extraction. The experimental drug was purchased from Jiangzhong Traditional Chinese Medicine Decoction Pieces Co., Ltd. It was identified as the aerial part of the labiform plant Elsholtzia MCM, and the decoction was prepared by the pharmacy department of the affiliated hospital, and the liquid equivalent of the dry herb is $1.5: 1$.

2.3. Experimental Apparatus. The following instruments were used: automatic blood coagulation analyzer (Japan Sysmex Corporation, version: Japan Sysmex, CA-1500), automatic biochemical analyzer (American Beckman Coulter, version: Beckman Coulter AU480), inverted microscope (Japan Nikon, version: TS100-F), electronic balance (Ihaus/Shanghai version: CP114), multifunctional microplate reader (Thermofidel Technology Co., Ltd. Version: Varioskan Flash), high-speed refrigerated centrifuge (Shanghai Sai Murphysal Technology Co., Ltd.), UltiMate 3000 ultra-high performance liquid chromatography system, and LTQ Orbitrap Velos Pro high-resolution mass spectrometer instrument (Thermo Fisher Company).

2.4. Experimental Reagents. The following were used: ALT (alanine transaminase), AST (aspartate aminotransferase), ALP (alkaline phosphatase), GGT (glutamyl transferase), TB (total bilirubin), DB (direct bilirubin), TP (total protein), ALB (albumin), CHE (cholinesterase), TBA (total bile acid), UA (uric acid), TG (triglyceride), TC (total cholesterol),
HDL (high-density lipoprotein), LDL (low-density lipoprotein), GLU (glucose), ADA (adenosine deaminase), CRE (creatinine), BUN (blood urea nitrogen) level detection kit (Shanghai Kehua Biotechnology Co., Ltd.), MDA (malondialdehyde), LPO (lipid peroxide), TNF (tumor necrosis factor), IL-1 (interleukin1), GAS (gastrin), Mtl (motilin), Na+-K+ATP, CGRP (calcitonin gene-related peptide), ET-1 (endothelin1) level of blood detection kit (provided by Shanghai Hepai Biotechnology Co., Ltd.). HPLC grade methanol and acetonitrile were purchased from Anaqua (Wilmington, DE USA). Distilled water was obtained from a Milli-Q Ultrapure water system (Millipore, Billerica, MA, USA).

2.5. Animal Exposure. Eighty SD rats, half male and half female, were randomly divided into eight groups, consisting of a normal control group (C), $0.2 \mathrm{~g} / \mathrm{kg} /$ day group (L), $2 \mathrm{~g} / \mathrm{kg} /$ day group $(\mathrm{M})$, and $20 \mathrm{~g} / \mathrm{kg} /$ day group $(\mathrm{H})$. Thus, each group consisted of 20 rats. All rats were acclimatized for one week and given normal food and distilled water. The treatment was conducted according to the following procedures. In the normal control group, animals were administered normal food and equal amount of distilled water without MCM water extract. Water MCM extract at doses of $0.2,2$, and $20 \mathrm{~g} / \mathrm{kg} /$ day was given to the $\mathrm{L}, \mathrm{M}$, and $\mathrm{H}$ groups, respectively. The entire administration period was 90 days. It was administered per os once a day $(1 \mathrm{ml} / 100 \mathrm{~g}$ b.w.). The body weight of the rats was recorded once a week, and the dose of MCM was adjusted according to the new body weight. At the beginning of the experiment, the difference in animal weight was not over or under $10 \%$ of the average weight.

\subsection{Observation Indicators}

2.6.1. Observation of Clinical Manifestations. We observe the clinical manifestations of rats in the cage after gavage every day, including health status, feeding and drinking behaviors, food intake, and the color of the hair and fur.

2.6.2. Biochemistry Analysis. At the end of the experiment, blood routine and liver and kidney biochemical function tests were done. Rats were anesthetized with $2 \%$ pentobarbital sodium, and blood samples were collected from the femoral artery for hematology and blood biochemical examination.

2.6.3. Determination of Immunology, Gastrointestinal Motility, Antioxidation, and Energy Metabolic Capacity. The levels of rat serum immunoglobulin (IgA, IgM, and IgG) and complement ( $\mathrm{C} 3$ and $\mathrm{C} 4)$ were measured by detection kits. The intestinal kinetics, oxidation, and energy metabolism indexes of $\mathrm{Na}^{+}-\mathrm{K}^{+}$-ATP, MDA, LPO, TNF, IL-1, GAS, Mtl, CGRP, and ET- 1 were measured by ELISA assay.

2.6.4. Histological Examination. Rats were dissected after blood collection, and organs including the heart, liver, spleen, lung, kidney, stomach, thymus, pancreas, adrenal gland, brain, male testis epididymis, female ovary, and uterus were collected and weighed. Then, they were fixed and stored with $4 \%$ formaldehyde solution. All organs were washed and dehydrated, paraffin embedded, sectioned, stained with hematoxylin- 


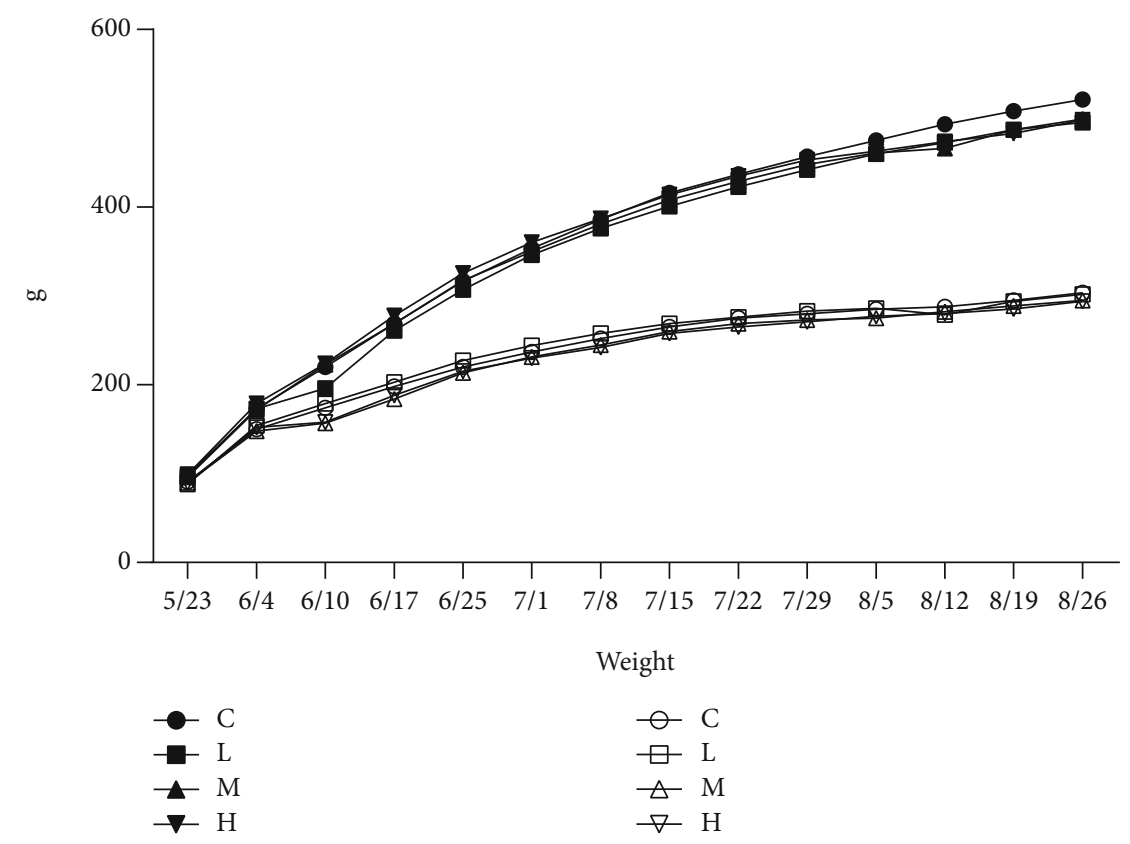

FIGURE 1: Effect of MCM on body weight in rats $(n=10)$. The hollow shapes represent female rat groups and solid shapes represent male rat groups. C: normal control group; L: water extraction of MCM $0.2 \mathrm{~g} / \mathrm{kg} / \mathrm{day}$; M: water extraction of MCM $2 \mathrm{~g} / \mathrm{kg} / \mathrm{day}$; H: water extraction of MCM $20 \mathrm{~g} / \mathrm{kg} /$ day.
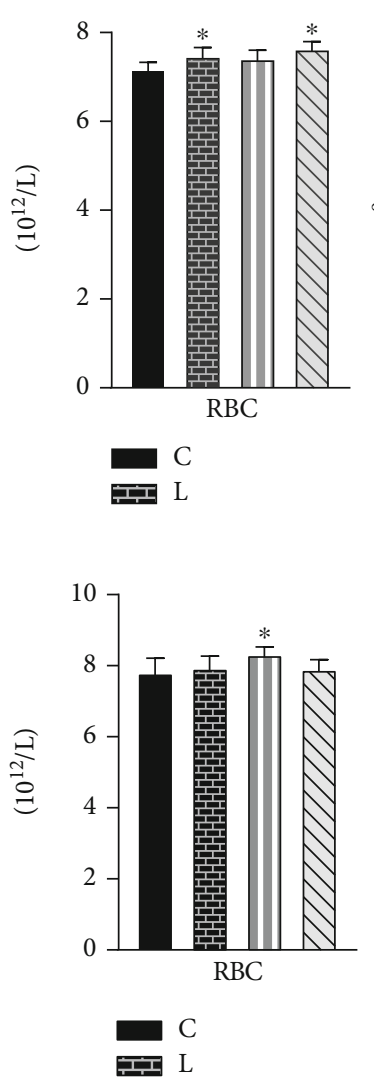

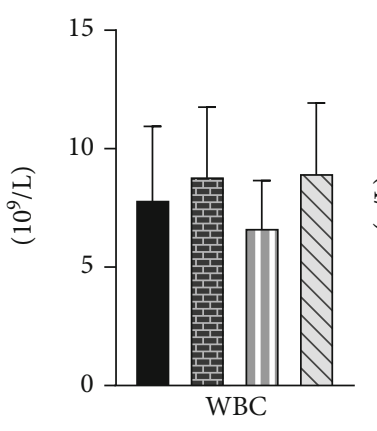

$\square$
$\Delta \nabla$

(a)
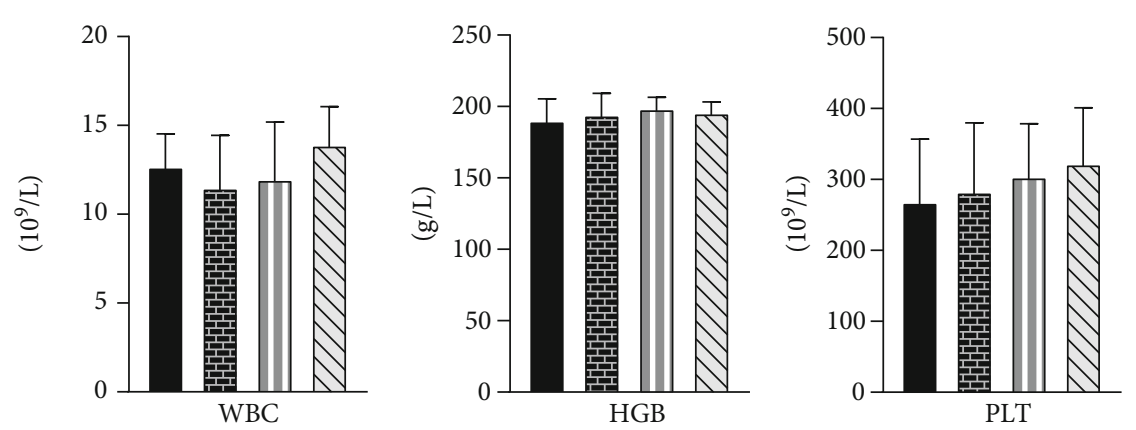

(b)

FIGURE 2: Effect of MCM on routine blood in rats. (a) represents female rat groups and (b) represents male rat groups. C: normal control group; L: water extraction of MCM $0.2 \mathrm{~g} / \mathrm{kg} /$ day; M: water extraction of MCM $2 \mathrm{~g} / \mathrm{kg} / \mathrm{day}$; H: water extraction of MCM $20 \mathrm{~g} / \mathrm{kg} /$ day.c ${ }^{*} P<$ 0.05 , compared with the control group; ${ }^{* *} P<0.01$, compared with the control group. MCM: Mosla chinensis Maxim; RBC: red blood cell; WBC: white blood cell; HGB: hemoglobin; PLT: platelet. 


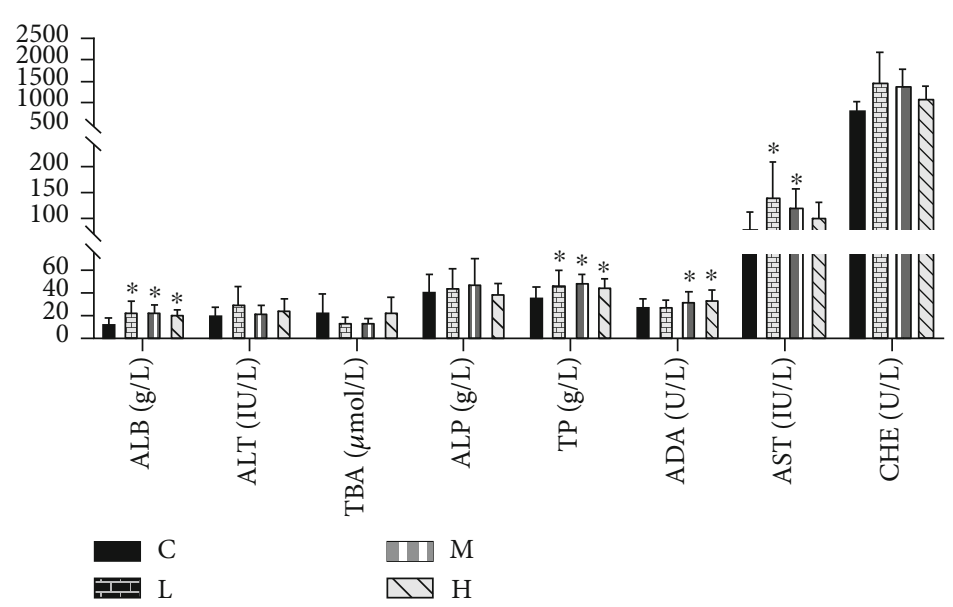

(a)

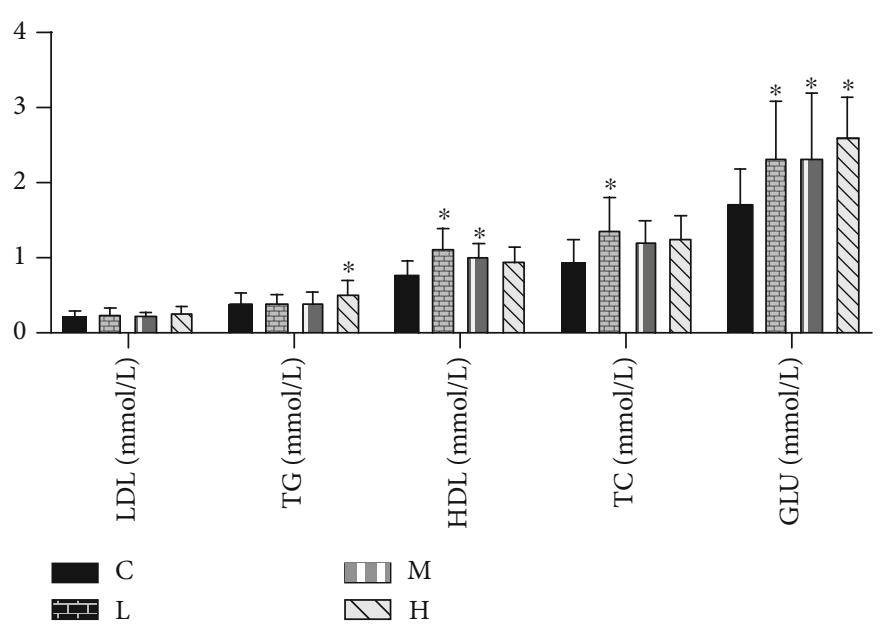

(c)

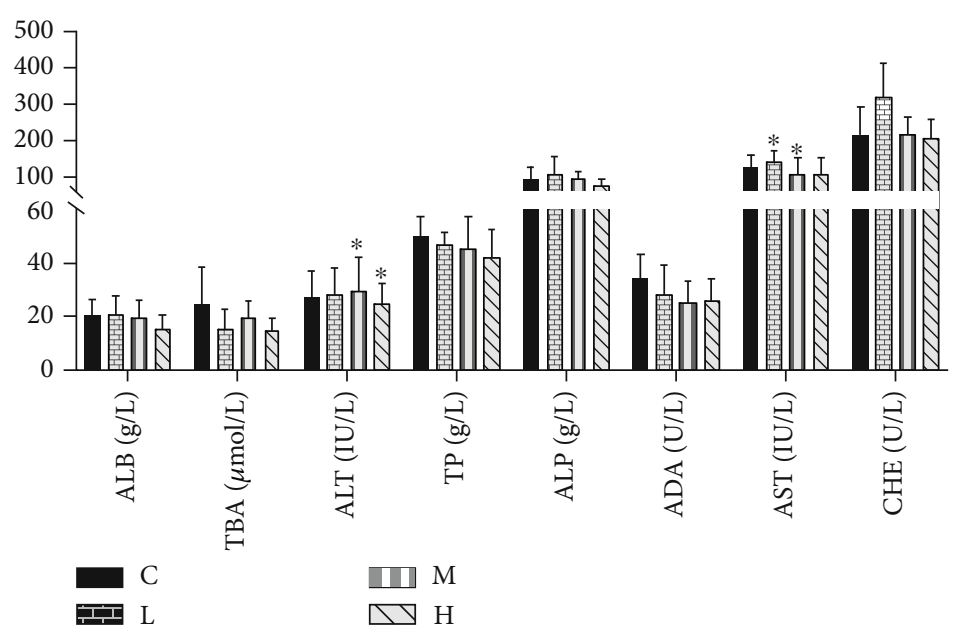

(e)

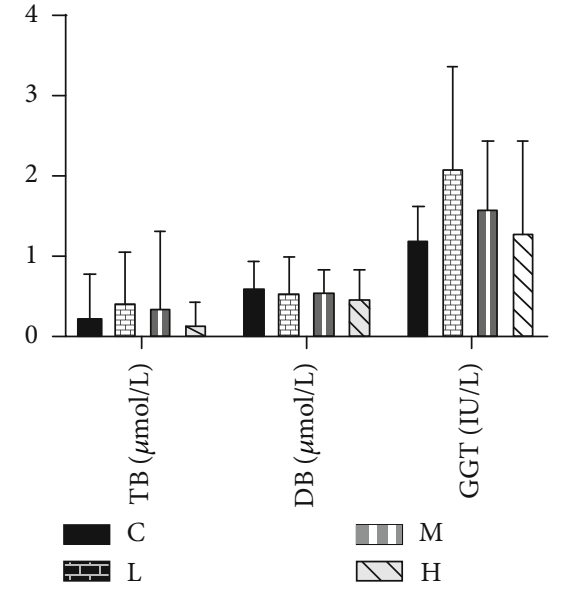

(b)

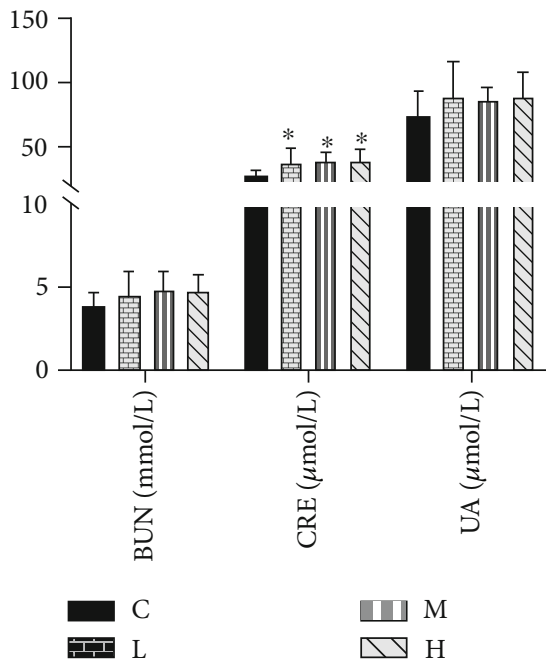

(d)

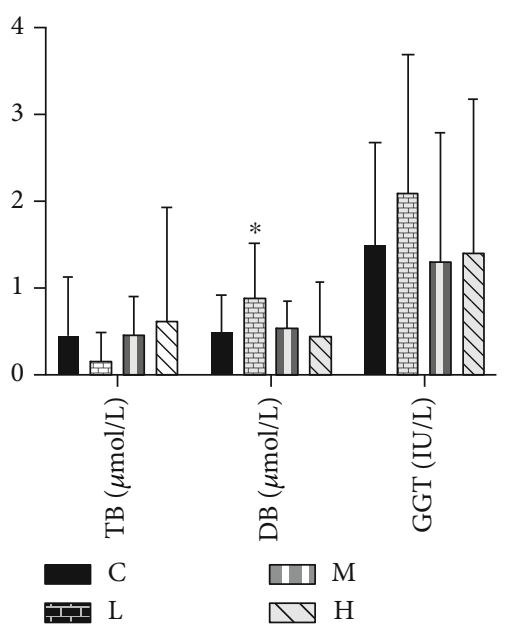

(f)

Figure 3: Continued. 


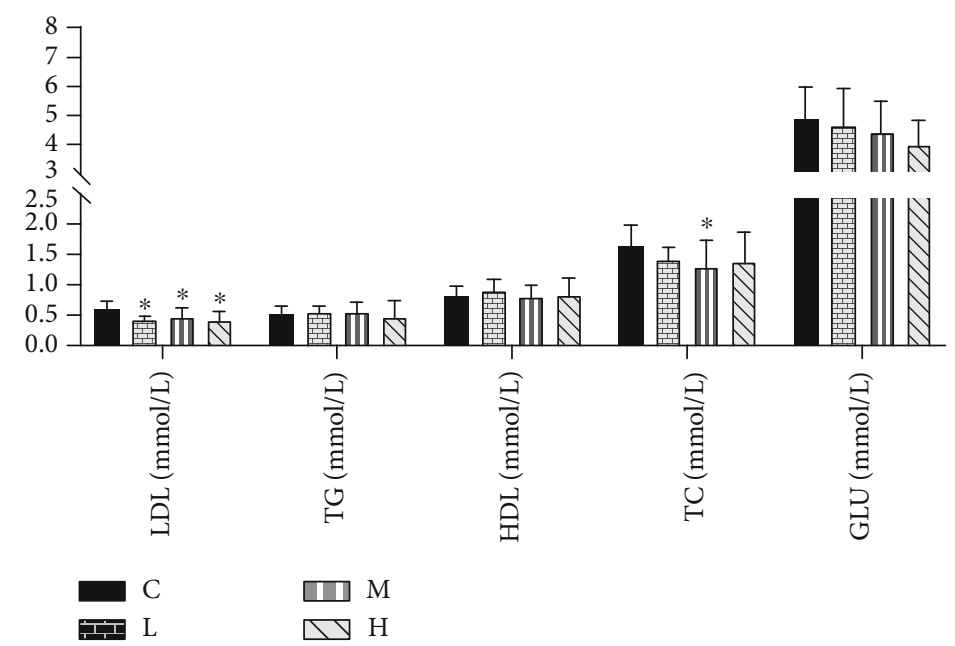

(g)

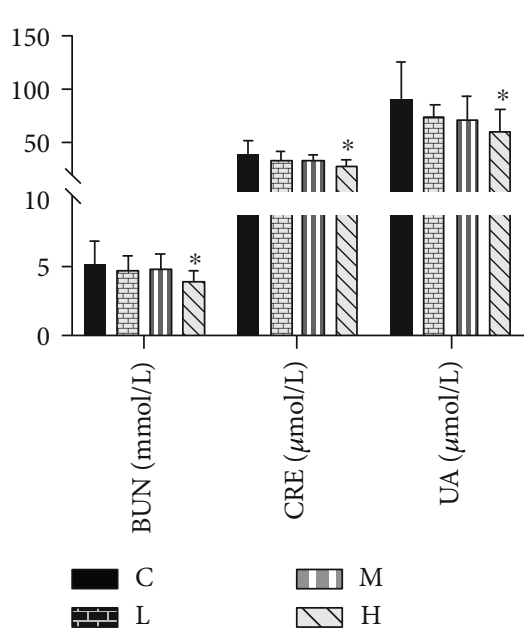

(h)

FIgURE 3: Effect of MCM on liver and kidney function-related indexes, blood glucose, and blood lipids in rats. (a-d) represent female rat groups and $(\mathrm{e}-\mathrm{h})$ represent male rat groups. C: normal control group; L: water extraction of MCM $0.2 \mathrm{~g} / \mathrm{kg} / \mathrm{day}$; M: water extraction of MCM $2 \mathrm{~g} / \mathrm{kg} /$ day; $\mathrm{H}$ : water extraction of MCM $20 \mathrm{~g} / \mathrm{kg} /$ day. ${ }^{*} P<0.05$, compared with the control group; ${ }^{* *} P<0.01$, compared with the control group. ALB: albumin; TBA: total bile acid; ALT: alanine transaminase; TP: total protein; ALP: alkaline phosphatase; ADA: adenosine deaminase; AST: aspartate aminotransferase; CHE: cholinesterase; TB: total bilirubin; DB: direct bilirubin; GGT: glutamyl transferase; LDL: low-density lipoprotein; TG: triglyceride; TC: total cholesterol; HDL: high-density lipoprotein; GLU: glucose; BUN: blood urea nitrogen; CRE: creatinine; UA: uric acid.

eosin (HE), and observed under an optical microscope, and routine pathological examinations were performed.

2.6.5. Determination of Intestinal Flora. Rats were fasted on the day before dissection, and feces were collected from the anus by tail-lifting stimulation, placed in a cryotube, and frozen at $-80^{\circ} \mathrm{C}$, and the sequence examination of intestinal flora was conducted in the Shanghai Majorbio Bio-pharm Technology Co., Ltd.

2.6.6. Component Identification of Urine. UHPLC analysis was performed on an Ultimate 3000 UHPLC system (Thermo Scientific, San Jose CA, USA), and the chromatographic separation was carried out using ACQUITY UPLC BEH C18 $1.7 \mu \mathrm{m}$ column $(2.1 \times 100 \mathrm{~mm}$, Waters Corporation, Ireland). The UHPLC mobile phase consisted of $0.1 \%$ aqueous formic acid (solvent A) and acetonitrile (solvent B). The gradient duration is presented in Table S1. The column temperature was kept constant at $40^{\circ} \mathrm{C}$. A blank sample is applied between every ten samples to wash the column.

An orbitrap mass spectrometer (LTQ Orbitrap Velos Pro, Thermo Fisher Scientific, San Jose, CA, USA) equipped with a heated electrospray ionization (HESI) probe was coupled to the UHPLC system. Detection was performed in both positive and negative ionization. The resolution type was set to 60000 FWHM, and all samples were operated on under the full scan mode. Dynamic exclusion was set to exclude a precursor ion for repeated MS/MS analysis within $15 \mathrm{~s}$. The activation type was collision-induced dissociation (CID), and the intensity threshold was set at 1000 . Other ion-source parameters of MS experiments were set as follows: heater temp, $350^{\circ} \mathrm{C}$; sheath gas flow rate, $35 \mathrm{arb}$; aux gas flow rate, $10 \mathrm{arb}$; sweep gas flow rate, $0 \mathrm{arb}$; I spray voltage, $3.6 \mathrm{kV}$; capillary temp, $320^{\circ} \mathrm{C}$; mass range, $\mathrm{m} / \mathrm{z} 100-1000$.

2.7. Statistical Analysis. Data were tested for significance between groups using IBM SPSS 26.0 application software, and single-factor ANOVA was used to test body weight, blood routine, blood biochemical indicators, and ELISA data. The UPLC-MS data were analyzed using SIMCA (Umetrics Company, USA), and also, they were analyzed using principal component analysis (PCA) and partial least squares discriminant analysis (PLS-DA) for multivariate statistical analysis. PCA, an unsupervised multivariate statistical approach, can reduce the dimensionalities of complex datasets and provide an overview of all observations in data tables. PLS-DA is used to reveal the net treatment effect on the subjects to detect the ions that have the greatest effect on the variance. Potential biomarkers were explored on the basis of variable importance in the project (VIP $>1$ ) value. VIP value of PLS-DA plays a major role in separation of the factors. The one-way analysis of variance (ANOVA) was applied to measure the significance of each metabolite. Metabolites with both multivariate statistical significance $(\mathrm{VIP}>1)$ and univariate statistical significance $(P<0.05)$ were considered to be markers responsible for the differentiation of the MCM treatment group from the control group. Finally, the ion spectrum of potential biomarkers was matched with the structure message of metabolites acquired from the Human Metabolome Database HMDB. Also, please refer to the following databases: METLIN (http://metlin .scripps.edu/), KEGG (http://www.kegg.com/), and mzCloud (https://www.mzcloud.org/). GraphPad Prism 8.0.2 application software was for graphing software. 


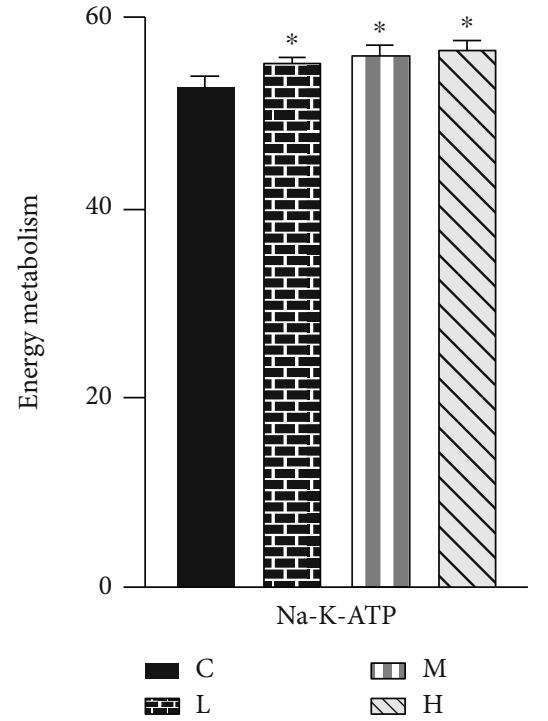

(a)

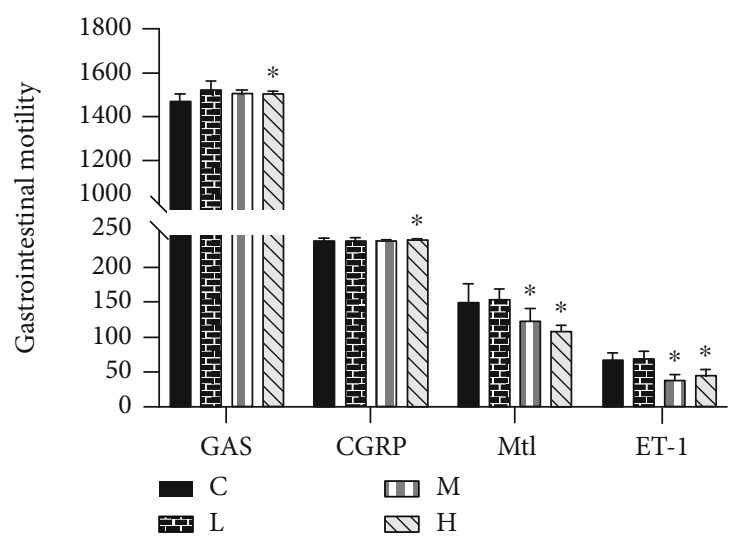

(c)

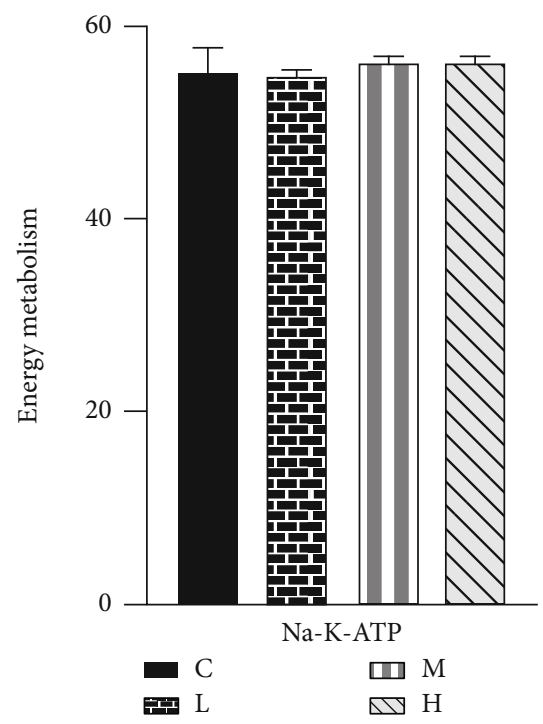

(e)

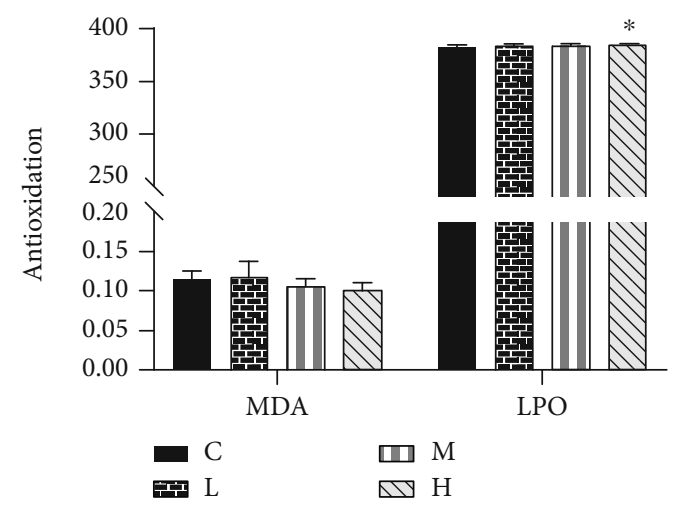

(b)

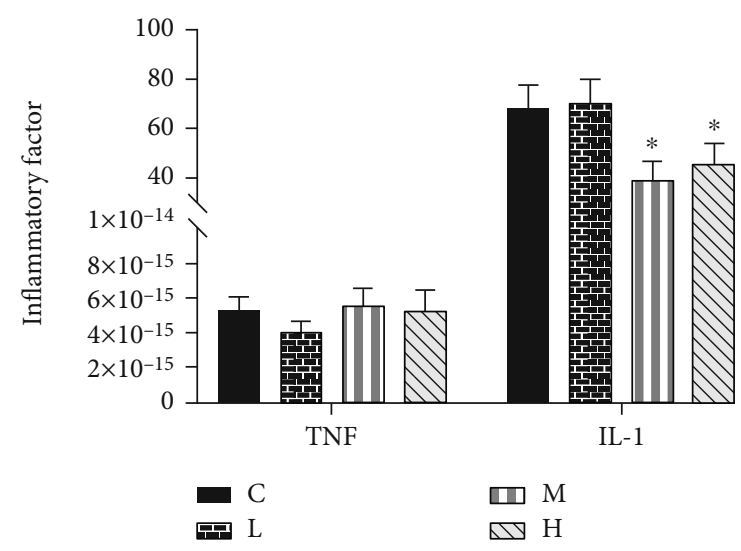

(d)

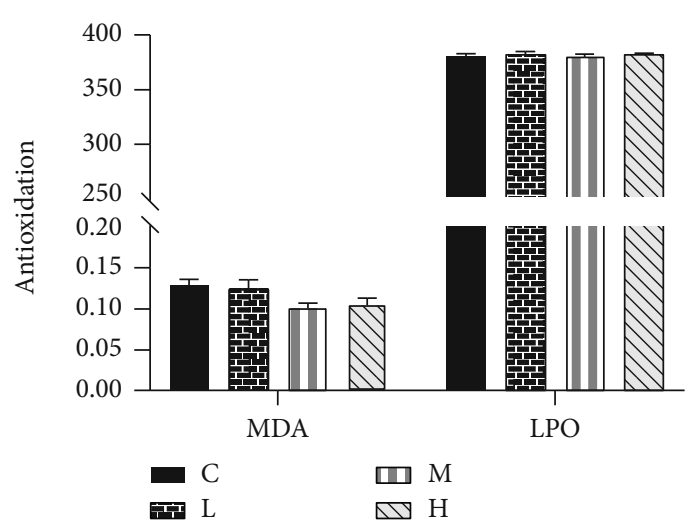

(f)

Figure 4: Continued. 


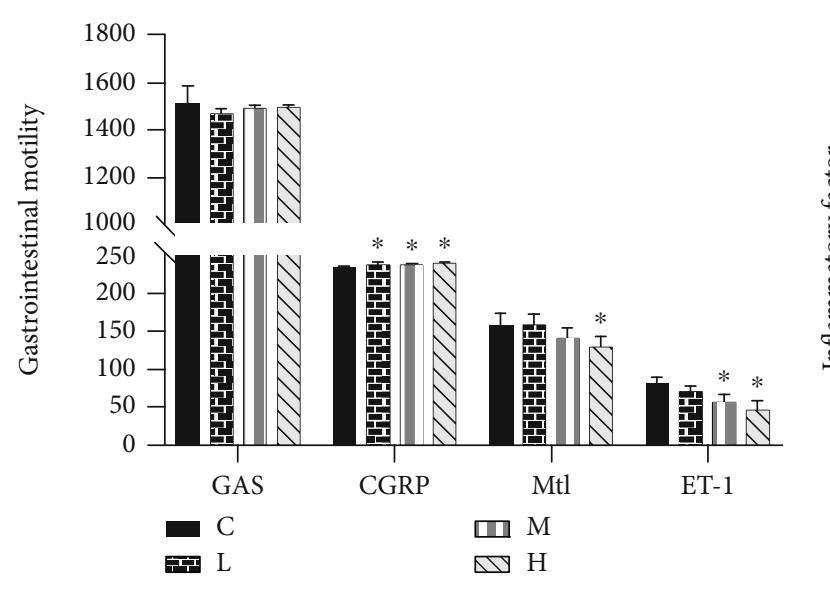

(g)

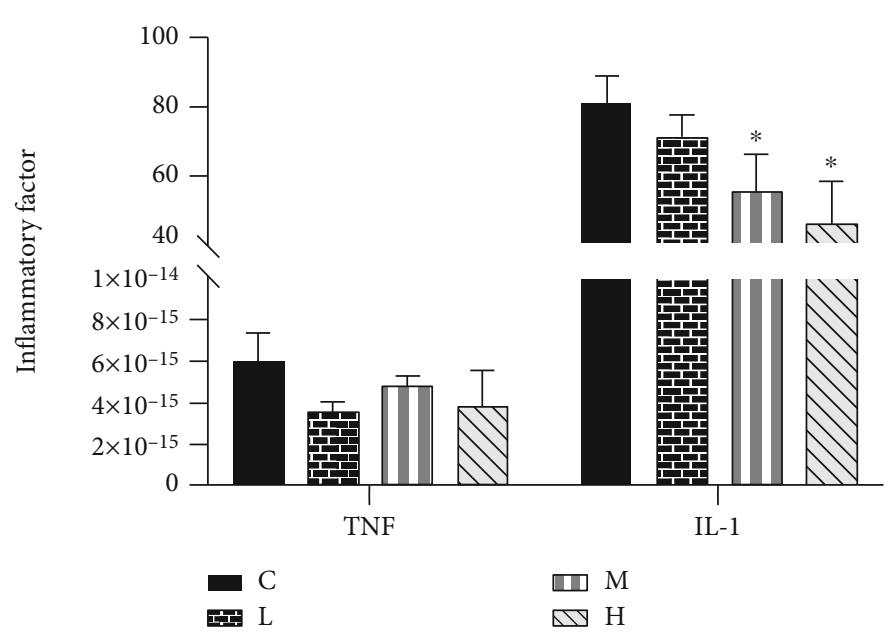

(h)

FIGURE 4: Effect of MCM on energy metabolism, oxidation, and gastrointestinal motility in rats. C: normal control group; L: water extraction of MCM $0.2 \mathrm{~g} / \mathrm{kg} /$ day; M: water extraction of MCM $2 \mathrm{~g} / \mathrm{kg} /$ day; $\mathrm{H}$ : water extraction of MCM $20 \mathrm{~g} / \mathrm{kg} / \mathrm{day} .{ }^{*} P<0.05$, compared with the control group; ${ }^{* *} P<0.01$, compared with the control group. MDA: malondialdehyde; LPO: lipid peroxide; GAS: gastrin; CGRP: calcitonin gene-related peptide; Mtl: motilin; ET-1: endothelin1; TNF: tumor necrosis factor; IL-1: interleukin1.

\section{Results}

3.1. Effects of MCM Administration on Clinical Manifestations. The results showed that there were no significant physical toxicity signs and symptoms in behavior, activity, hair and fur color, food intake, drinking water, external reaction, and feces between the experimental group and the normal control group.

3.2. Effects of MCM Administration on Body Weight. As shown in Figure 1, the body weight of all groups increased with the feeding time, and there was no obvious difference between the experimental groups and the normal control group $(P>0.05)$.

3.3. Effects of MCM Administration on Routine Blood Analysis. The blood routine test results of rats, after 90 days of administration, are shown in Figure 2. The numbers of red blood cells in the 0.2 and $20 \mathrm{~g} / \mathrm{kg} /$ day female groups significantly increased compared with the female control group $(P<0.05)$ (Figure $2(\mathrm{a})$ ), and the male rats in the $2 \mathrm{~g} / \mathrm{kg} / \mathrm{day}$ group were significantly increased compared with those in the male control group (Figure $2(\mathrm{~b}))(P<0.05)$. No significant abnormalities were found in the number of other cells.

3.4. Effect of MCM on Serum Biochemical Index. After the end of administration, serum was collected from each rat for liver and kidney biochemical index detection, and the results are shown in Figure 3. The levels of ALT, GGT, $\mathrm{ALP}, \mathrm{TB}$, and $\mathrm{DB}$ of female rats in the experimental group were not significantly different from those in the control group. The AST level which increased after administration in all 0.2 and $2 \mathrm{~g} / \mathrm{kg} /$ day groups was significantly higher than that in the control group $(P<0.05)$, and with the increase of the dosage, there is a decreasing trend (Figures 3(a) and 3(e)). The levels of TP, ALB, and ADA in all experimental groups were higher than those in the control group, and there was a significant difference of TP and ALB levels compared with the control group $(P<0.05)$.

The results of blood glucose and blood lipid parameters of female rats showed that there was no significant difference in LDL in the administration group compared with the control group; the levels of GLU, TG, and TC were all higher than in the control group; TG levels in the $20 \mathrm{~g} / \mathrm{kg} /$ day group and TC levels in the $0.2 \mathrm{~g} / \mathrm{kg} /$ day group were significantly different from those in the control group; HDL levels in the 0.2 and $2 \mathrm{~g} / \mathrm{kg} /$ day groups were significantly higher compared with those in the control group $(P<0.05)$; and the levels of GLU in the $0.2,2$, and $20 \mathrm{~g} / \mathrm{kg} /$ day groups were significantly different from those in the control group $(P<0.05)$, as shown in Figure 3(c). The levels of BUN and UA in the female administration group were no different from the control group, but the levels of CRE were significantly higher than those in the control group $(P<0.05)$, as shown in Figure 3(d). However, the results of the blood glucose and blood lipid parameters of male rats showed that there were no significant differences between the groups, as shown in Figure 3.

3.5. Effect of MCM on Energy Metabolism, Oxidation, and Gastrointestinal Motility. The oxidation and energy metabolism indexes of rats were measured by ELISA assay, and the results are shown in Figures $4(\mathrm{a})-4(\mathrm{~d})$ (female rats) and Figures 4(e)-4(h) (male rats). For energy metabolism, the $\mathrm{Na}^{+}-\mathrm{K}^{+}$-ATP level in the serum of all experimental groups was higher than that of the control group and showed a certain dose-response relationship, but only the female experimental group showed a significant difference $(P<0.05)$. In terms of antioxidant capacity, MDA level of the administration group was not significantly different from that of the control group, but LPO level of the female $20 \mathrm{~g} / \mathrm{kg} /$ day group was significantly different from that of the control group $(P<0.05)$. For gastrointestinal motility, Mtl and ET-1 levels in all 2 and $20 \mathrm{~g} / \mathrm{kg} /$ day groups were significantly lower than those in the control group $(P<0.05)$, 

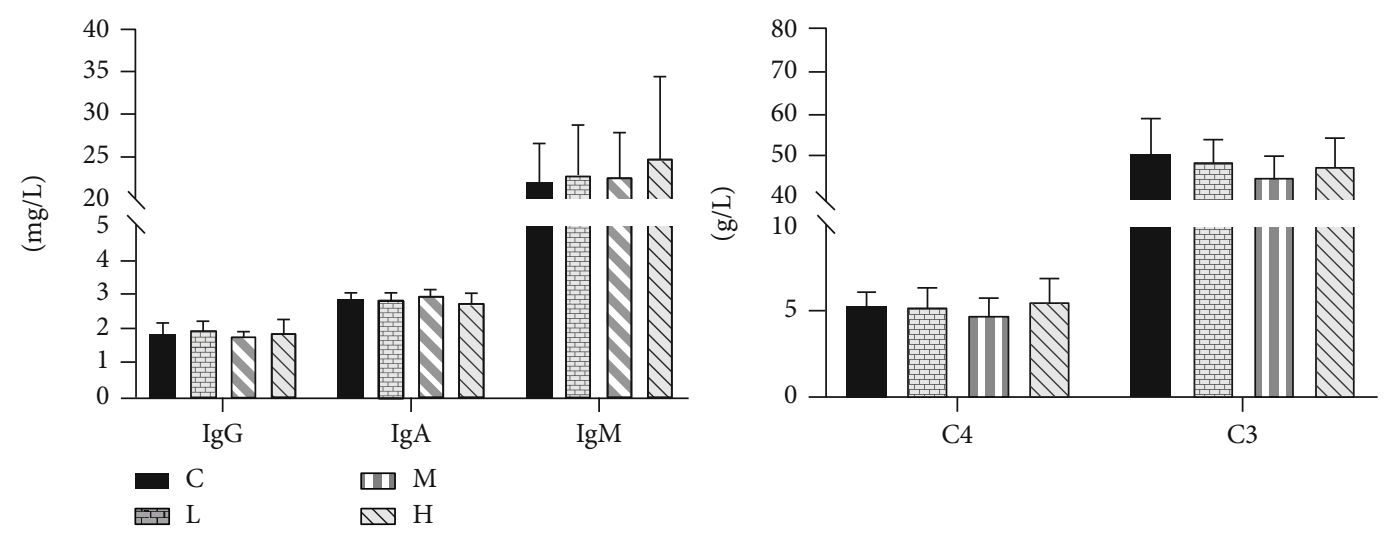

(a)
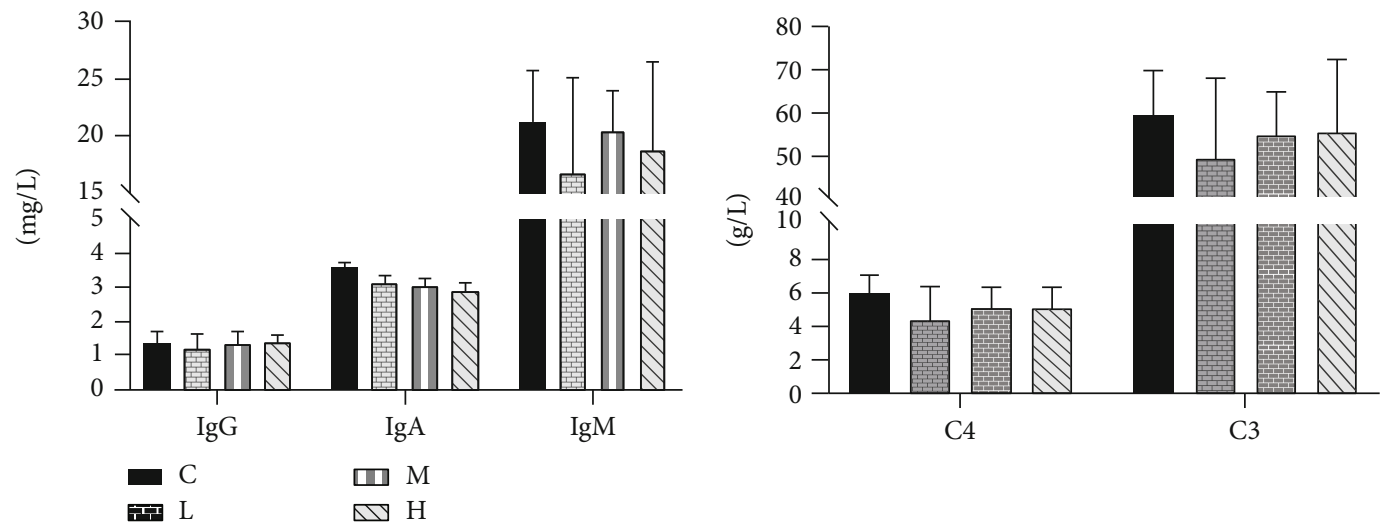

(b)

FIGURE 5: Effect of MCM on immunity in rats. C: normal control group; L: water extraction of MCM 0.2 g/kg/day; M: water extraction of MCM $2 \mathrm{~g} / \mathrm{kg} /$ day; $\mathrm{H}$ : water extraction of MCM $20 \mathrm{~g} / \mathrm{kg} /$ day. ${ }^{*} \mathrm{P}<0.05$, compared with the control group; ${ }^{* *} P<0.01$, compared with the control group. IgG: immunoglobulin G; IgA: immunoglobulin A; IgM: immunoglobulin M; C4: complement 4; C3: complement 3.

TABLE 1: Effect of MCM on urine routine in rats.

\begin{tabular}{|c|c|c|c|c|c|c|c|c|}
\hline \multirow[b]{2}{*}{ Index } & \multicolumn{4}{|c|}{0} & \multicolumn{4}{|c|}{ 우 } \\
\hline & $\mathrm{C}$ & $\mathrm{L}$ & $\mathrm{M}$ & $\mathrm{H}$ & $\mathrm{C}$ & $\mathrm{L}$ & M & $\mathrm{H}$ \\
\hline Occult blood & $2 / 10$ & $2 / 10$ & $0 / 10$ & $0 / 10$ & $2 / 10$ & $2 / 10$ & $1 / 10$ & $1 / 10$ \\
\hline Protein & $1 / 10$ & $1 / 10$ & $0 / 10$ & $0 / 10$ & $1 / 10$ & $1 / 10$ & $0 / 10$ & $1 / 10$ \\
\hline Bilirubin & $2 / 10$ & $1 / 10$ & $0 / 10$ & $1 / 10$ & $1 / 10$ & $1 / 10$ & $1 / 10$ & $1 / 10$ \\
\hline Urobilinogen & $<16$ & $<16$ & $<16$ & $<16$ & $<16$ & $<16$ & $<16$ & $<16$ \\
\hline Ketone body & $0 / 10$ & $0 / 10$ & $0 / 10$ & $0 / 10$ & $0 / 10$ & $0 / 10$ & $0 / 10$ & $0 / 10$ \\
\hline Leukocyte & $0 / 10$ & $0 / 10$ & $0 / 10$ & $0 / 10$ & $0 / 10$ & $0 / 10$ & $0 / 10$ & $0 / 10$ \\
\hline
\end{tabular}

C: normal control group; L: water extraction of MCM $0.2 \mathrm{~g} / \mathrm{kg} /$ day; M: water extraction of MCM $2 \mathrm{~g} / \mathrm{kg} / \mathrm{day}$; H: water extraction of MCM $20 \mathrm{~g} / \mathrm{kg} / \mathrm{day}$.

and CGRP levels in all male and female $20 \mathrm{~g} / \mathrm{kg}$ /day groups were significantly different from those in the control group $(P<0.05)$. For inflammation, IL-1 levels were significantly lower in all 2 and $20 \mathrm{~g} / \mathrm{kg} /$ day groups than in the control group.

3.6. Effects of MCM on Immunity. The results of the serum immunoglobulin level are shown in Figure 5. The levels of IgA, IgM, IgG, C3, and $\mathrm{C} 4$ did not show any difference $(P>0.05)$ compared to those in the control group.

3.7. Effects of MCM on Routine Urine Test. The results of routine urine tests are shown in Table 1, and there was no significant difference between the experimental groups and the control group.

3.8. Effects of MCM on Organ Coefficient. As shown in Figure 6, the organ coefficients of each administration group except the liver were not significantly different from those of the control group. Compared with the normal control group, the organ coefficient of the liver of female rats is slightly lower at 2 and $20 \mathrm{~g} / \mathrm{kg} /$ day (Figure 6(a)). The liver organ coefficient of male rats increases with the increase of the administered dose, which has a significant dose escalation effect, 


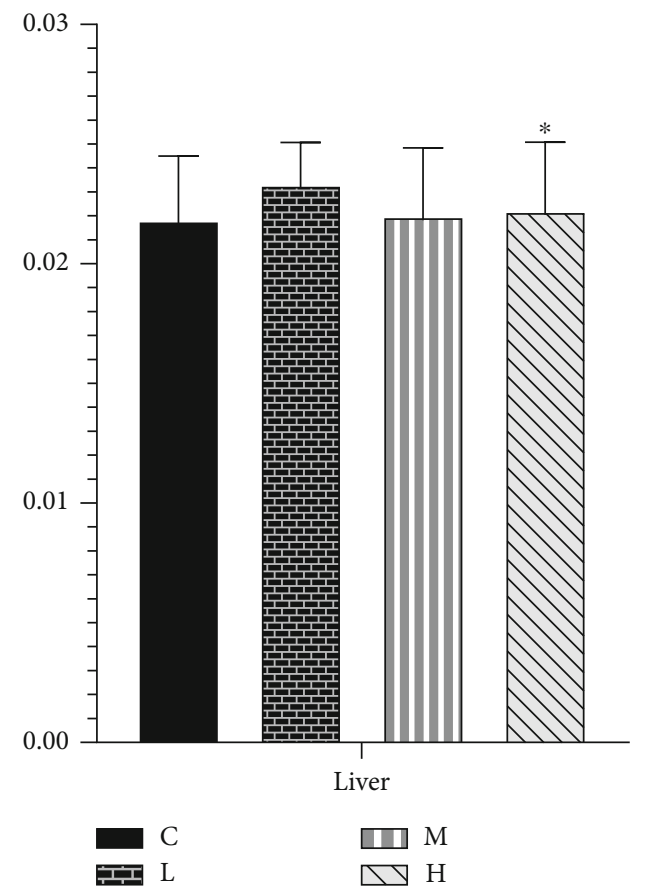

(a)

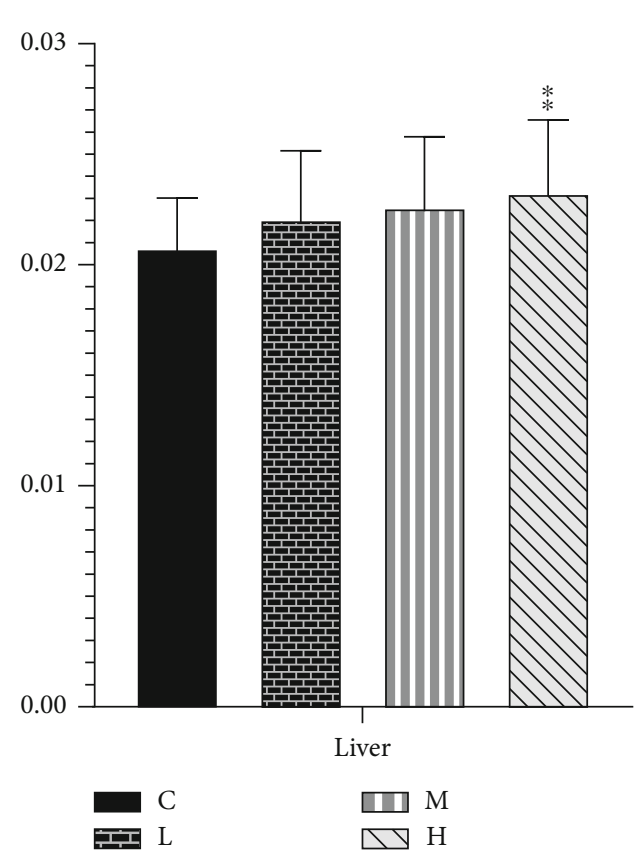

(b)

Figure 6: Effect of MCM on organ coefficient in rats $(n=10)$. C: normal control group; L: water extraction of MCM $0.2 \mathrm{~g} / \mathrm{kg} / \mathrm{day}$; M: water extraction of MCM $2 \mathrm{~g} / \mathrm{kg} /$ day; H: water extraction of MCM $20 \mathrm{~g} / \mathrm{kg} /$ day. ${ }^{*} P<0.05$, compared with the control group; ${ }^{* *} P<0.01$, compared with the control group.

and the $20 \mathrm{~g} / \mathrm{kg} /$ day group has a significant difference $(P<0.05)$ (Figure 6(b)).

\subsection{Effects of MCM on Histopathological Analysis. After 90} days, the rats were examined by routine autopsy; no obvious changes were observed by the naked eye. Rat liver, heart, spleen, lung, kidney, stomach, intestine, testis $\left({ }^{\star}\right)$, and uterus (O) tissues are used for routine histopathological examination. No abnormal histopathological changes were found in vital organs under an inverted microscope.

3.10. Effects of MCM on the Distribution of OTUs. We conducted $16 \mathrm{~S}$ rDNA sequencing to profile gut microbiota composition and used a Venn diagram to show shared and unique OTU numbers. For female rats, there were $527 \mathrm{com}$ mon OTUs, 7 unique OTUs in the control group, and 9, 9, and 5 unique OTUs in the $0.2,2$, and $20 \mathrm{~g} / \mathrm{kg} /$ day groups (Figure 7(a)), respectively. For male rats, there were $557 \mathrm{com}$ mon OTUs, while the unique OTUs in the control group were 7 and there were 5,12 , and 12 unique OTUs in the $0.2,2$, and $20 \mathrm{~g} / \mathrm{kg} / \mathrm{day}$ groups (Figure $7(\mathrm{~b})$ ), respectively.

As shown in Figure 8(a), MCM 90-day feeding changes the level of intestinal flora. Females have greater influence on Lactobacillus, Prevotella, Ruminococcus, Turicibacter, Romboutsia, and Alloprevotella relative abundance. Compared with the control group, the relative abundance of Romboutsia and Alloprevotella is significantly different $(P<0.05)$, as shown in Figure 8(b). The differences between the sunburst map sets of multiple species at the bacterial genera level are shown in Figure 9(c).
The difference in intestinal microflora after 90 days of feeding of MCM is shown in Figure 9, and the results show that MCM can change the number of intestinal flora (Figure 9(a)). In the female rat groups, MCM had a greater impact on lactobacillus, prevotella_9, ruminococeaceae_ucg_ 014, turicibacter, Romboutsia, and Alloprevotella, but the differences between the various genera did not reach a significant difference (Figure 9(b)); the difference between the sunburst diagram groups of multilevel species at the genus level is shown in Figure 9(c).

3.11. Effects of MCM on Urinary Metabolism. The urine samples of the control and treated groups were analyzed by applying UHPLC-LTQ-Orbitrap-MS/MS in both positive and negative ionization modes to confirm the significant metabolic alterations of metabonomic profiles. The typical base peak intensity chromatogram obtained from the analysis of the positive ionization mode of the urine samples is shown in Figure 10. The PCA plots for the treated groups clearly deviate from that of the control group by the 90th day (Figure 11). The more obvious the metabolic profile changes, the greater the influence of MCM.

The potential variables were selected as the biomarkers based on variable importance in projection (VIP) value $(>1)$ and ANOVA $(P<0.05)$. Finally, the 5 biomarkers $(2$ from the positive mode and 4 from the negative mode) were identified in urine (refer to Table 2) based on accurate mass, isotopic pattern, MS/MS information, and comparison with the structure message of metabolites. As shown in Table 2, indoleacetic acid, N-acetyl-L-phenylalanine, 2-hydroxycinnamic acid, kynurenic acid, and xanthurenic acid significantly 

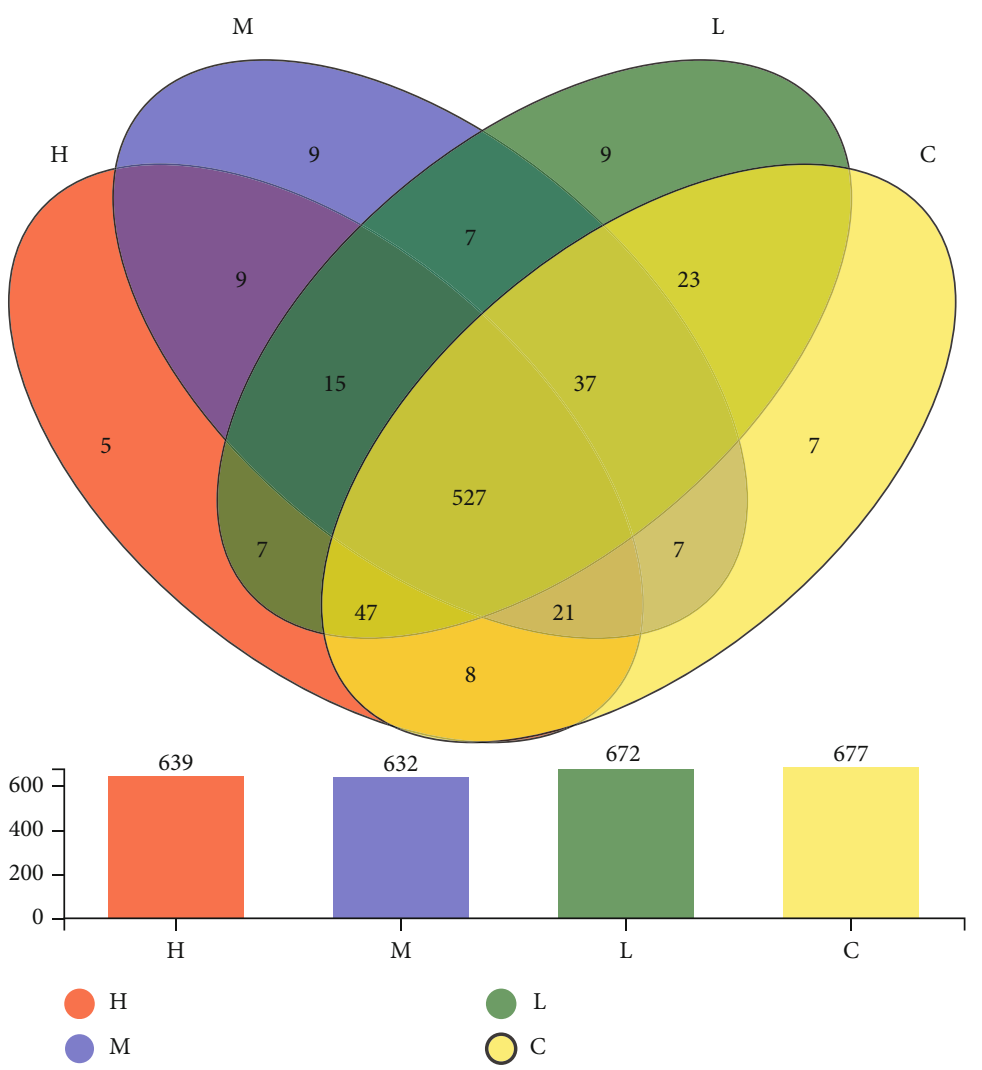

(a)

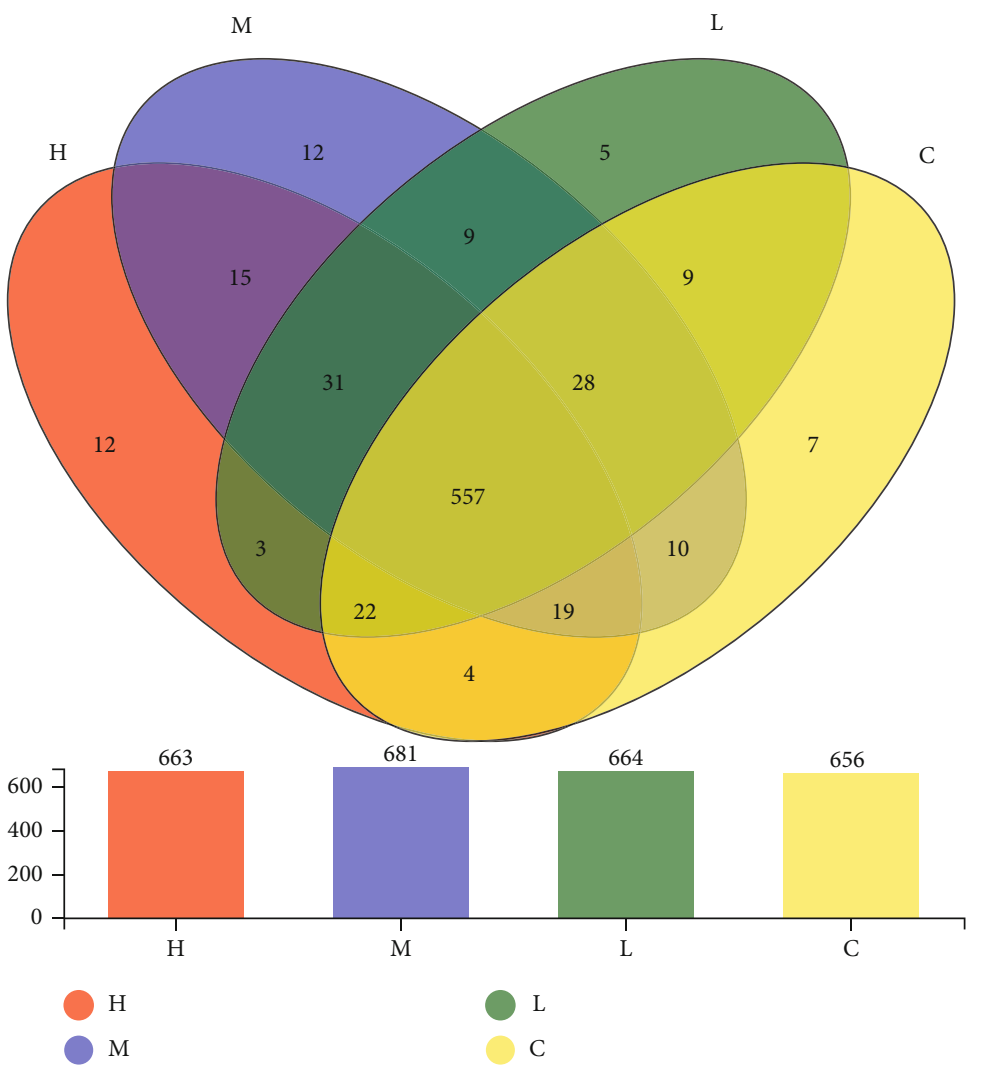

(b)

Figure 7: Effect of MCM on common/unique OTU number distribution in rats. C: normal control group; L: water extraction of MCM $0.2 \mathrm{~g} / \mathrm{kg} /$ day; M: water extraction of MCM $2 \mathrm{~g} / \mathrm{kg} /$ day; $\mathrm{H}$ : water extraction of MCM $20 \mathrm{~g} / \mathrm{kg} /$ day. 


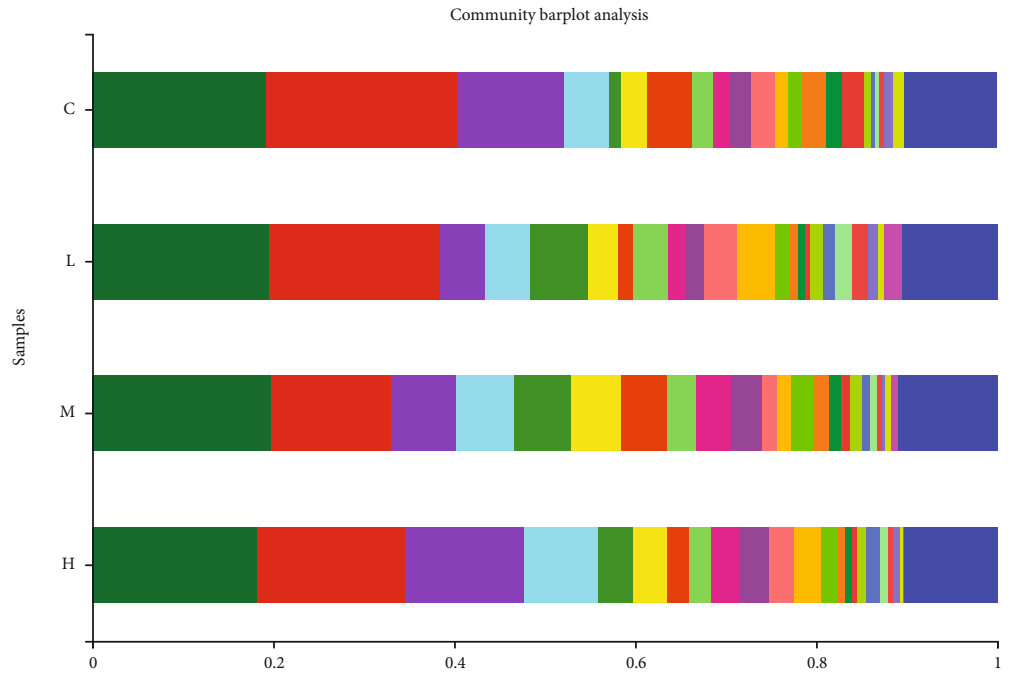

Percent of community abundance on Genus level

- Norank_f_Muribaculaceae

- Lactobacillus

Prevotella_9

Ruminococcaceae_UCG-014

- Ruminococcus_2

Romboutsia

Turicibacter

Ruminococcaceae_UCG-005

Prevotellaceae_UCG-001

Unclassified_f_Lachnospiraceae

Ruminococcus_1

Alloprevotella
Unclassified_f_Ruminococcaceae

Lachnospiraceae_NK4A136_gro

Ruminococcaceae_UCG-013

Chriniclostridium_6

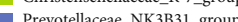

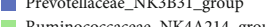

[Eubacterium]_coprostanoligenes_group

- Prevotellaceae_Ga6A1_group

[Eubacterium]_xylanophilum_group

Akkermansia

(a)

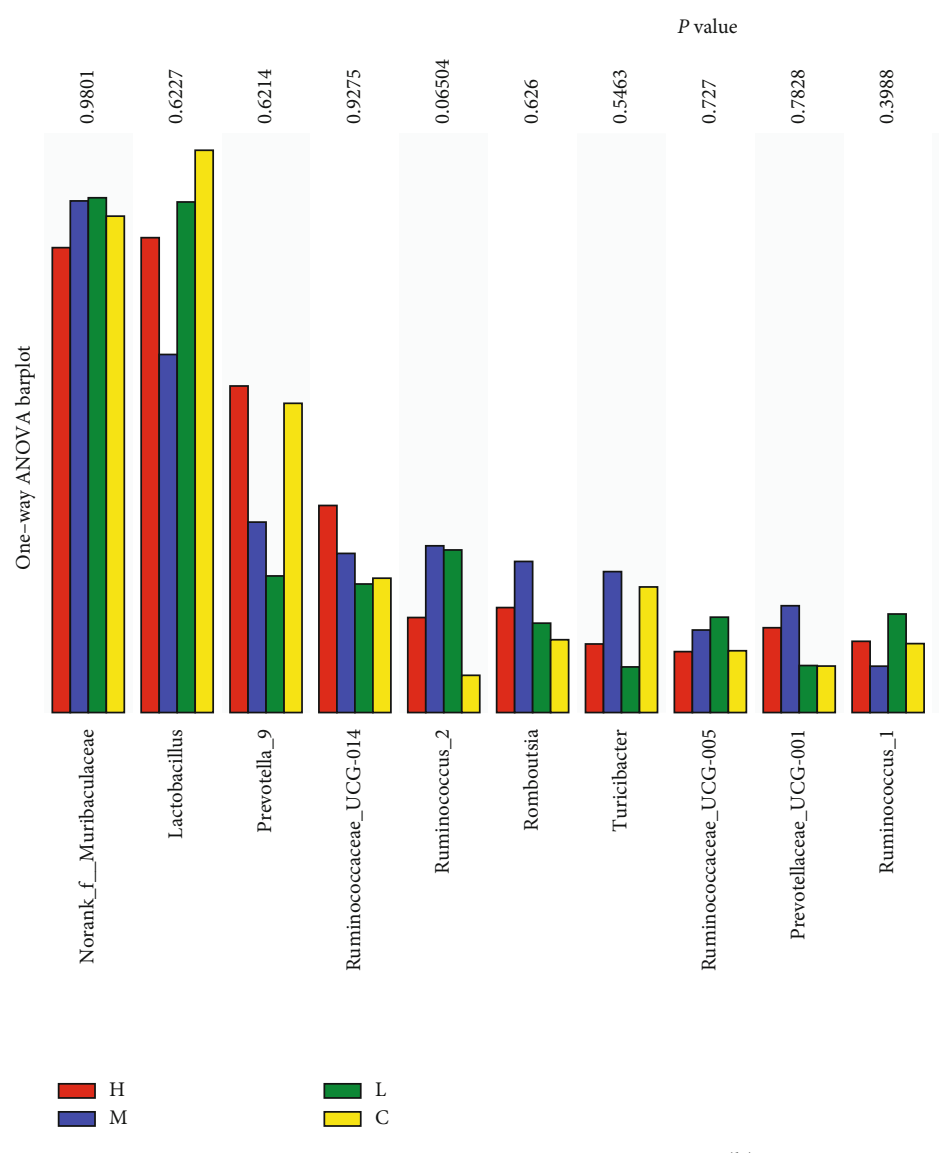

$\begin{array}{lllll}0 & 0 \\ 0 & 0 & 0 & 0 \\ 0 & 0 & 0 & 0 \\ 0 & 0 & 0 \\ 0 & 0 & 0 \\ 0 & 0 & 0\end{array}$

(b)

Figure 8: Continued. 

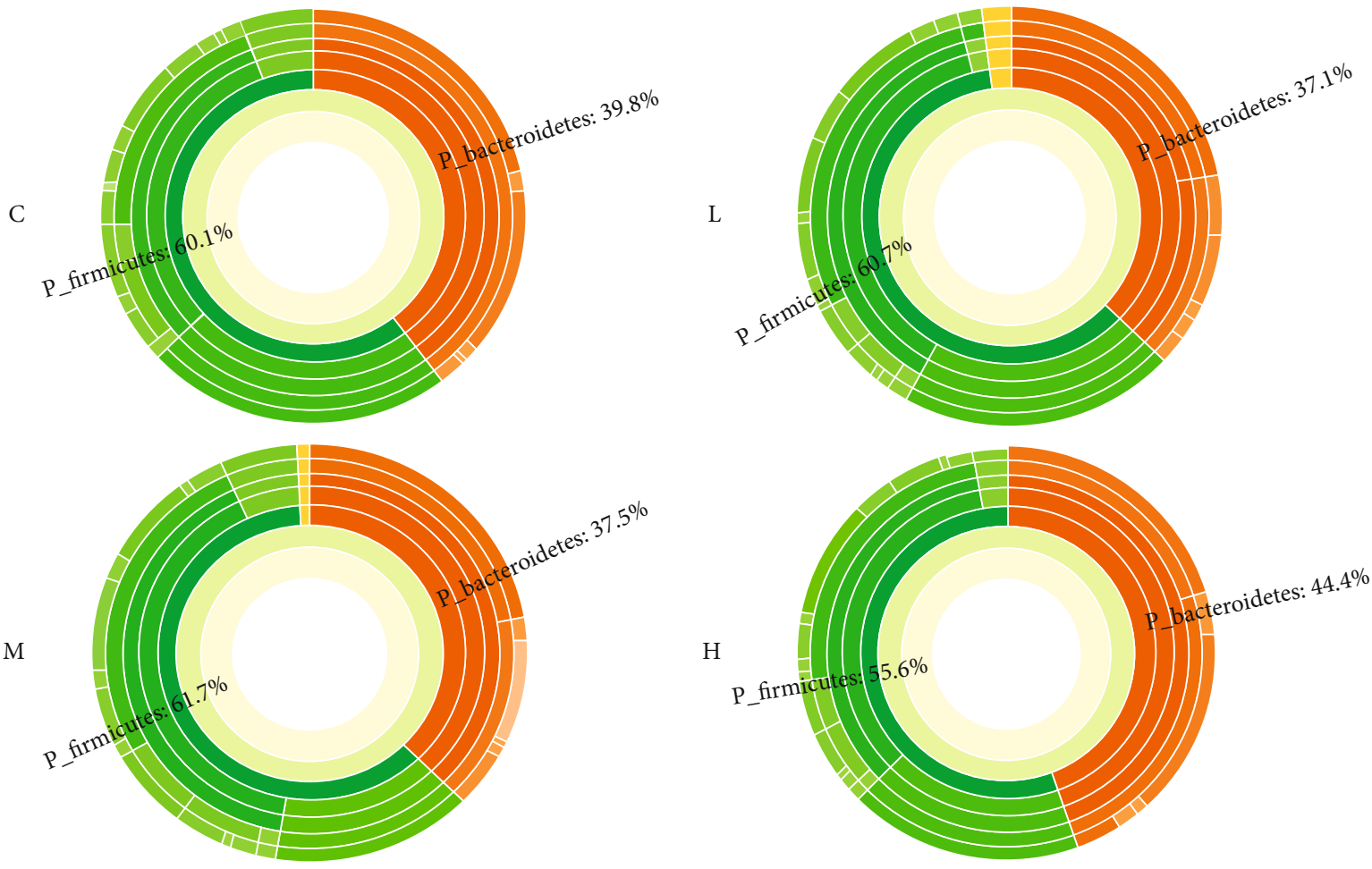

(c)

FIGURE 8: Effect of MCM on the relative abundance of gut microbiota in male rats. C: normal control group; L: water extraction of MCM $0.2 \mathrm{~g} / \mathrm{kg} /$ day; $\mathrm{M}$ : water extraction of MCM $2 \mathrm{~g} / \mathrm{kg} / \mathrm{day} ; \mathrm{H}$ : water extraction of MCM $20 \mathrm{~g} / \mathrm{kg} /$ day. ${ }^{*} P<0.05$, compared with the control group; ${ }^{* *} P<0.01$, compared with the control group.

decreased $(P<0.05)$. Furthermore, the urinary metabolites altered significantly in a dose-dependent manner. The 5 metabolites mentioned above demonstrated more significant changes in the $20 \mathrm{~g} / \mathrm{kg} /$ day group than in the $0.2 \mathrm{~g} / \mathrm{kg} / \mathrm{day}$ group. The metabolic profiling change in the $20 \mathrm{~g} / \mathrm{kg} /$ day group appeared to have more remarkable effects than that in the $2 \mathrm{~g} / \mathrm{kg} /$ day group.

\section{Discussion}

The use of traditional Chinese medicine has a long history in China. Understanding the toxicity of traditional Chinese medicine is the key to rational use of traditional Chinese medicine [5-7]. Modern pharmacological studies have found that some herbal ingredients of Aristolochiaceae are toxic [8]. For example, aristolochic acid in them shows relatively strong toxicity and even irreversible permanent carcinogenicity. In recent years, more and more attention has been paid to the evaluation of the safety of traditional Chinese medicine [9-12], and there are many new methods being explored, such as network pharmacology [13], genomics [14], comparative omics, and metabonomics [15-18]. The mammalian 90-day toxicity test is an important experiment for safety evaluation, and the traditional 90-day feeding test has relatively few end points and narrow coverage. Nowadays, some toxicologists have begun to explore the use of invertebrates as an alternative to mammals for long-term studies [19]. We added intestinal microbial diversity detection and urine mass spectrometry to the traditional 90-day feeding test analysis in order to obtain more comprehensive data for safety evaluation of subjects.

The weight of experimental animals during their growth and development is one of the most basic and sensitive indicators to comprehensively reflect the health status of animals [20], so it is an important observation indicator in the toxicity test, as well as the balance of lipid metabolism. In the 90day subchronic toxicity study of Elsholtzia Maxim, it was shown that it had no significant influence on the weight and growth of rats. However, the weight of male rats from the second week showed that the weight of the administration group was slightly lower than that of the normal control group, and the TC and LDL levels of the male administration group were significantly lower than those of the normal control group, and the HDL level increased. The results are in line with previous studies that MCM has the effect of lowering blood lipids [21].

The blood routine index is an important index to reflect whether the test substance has affected the hematopoietic function. The results of this experiment showed that the number of red blood cells in female rats with 0.2 and $20 \mathrm{~g} / \mathrm{kg} /$ day and male rats with $2 \mathrm{~g} / \mathrm{kg} /$ day increased after 90 days, but no obvious dose-effect relationship was observed, so comprehensive analysis showed that the feeding experiment of MCM for 90 days had no obvious influence on the blood routine of rats.

In order to improve the overall understanding of the safety of traditional Chinese medicine, we increased the detection of serum energy metabolism, oxidation index, 


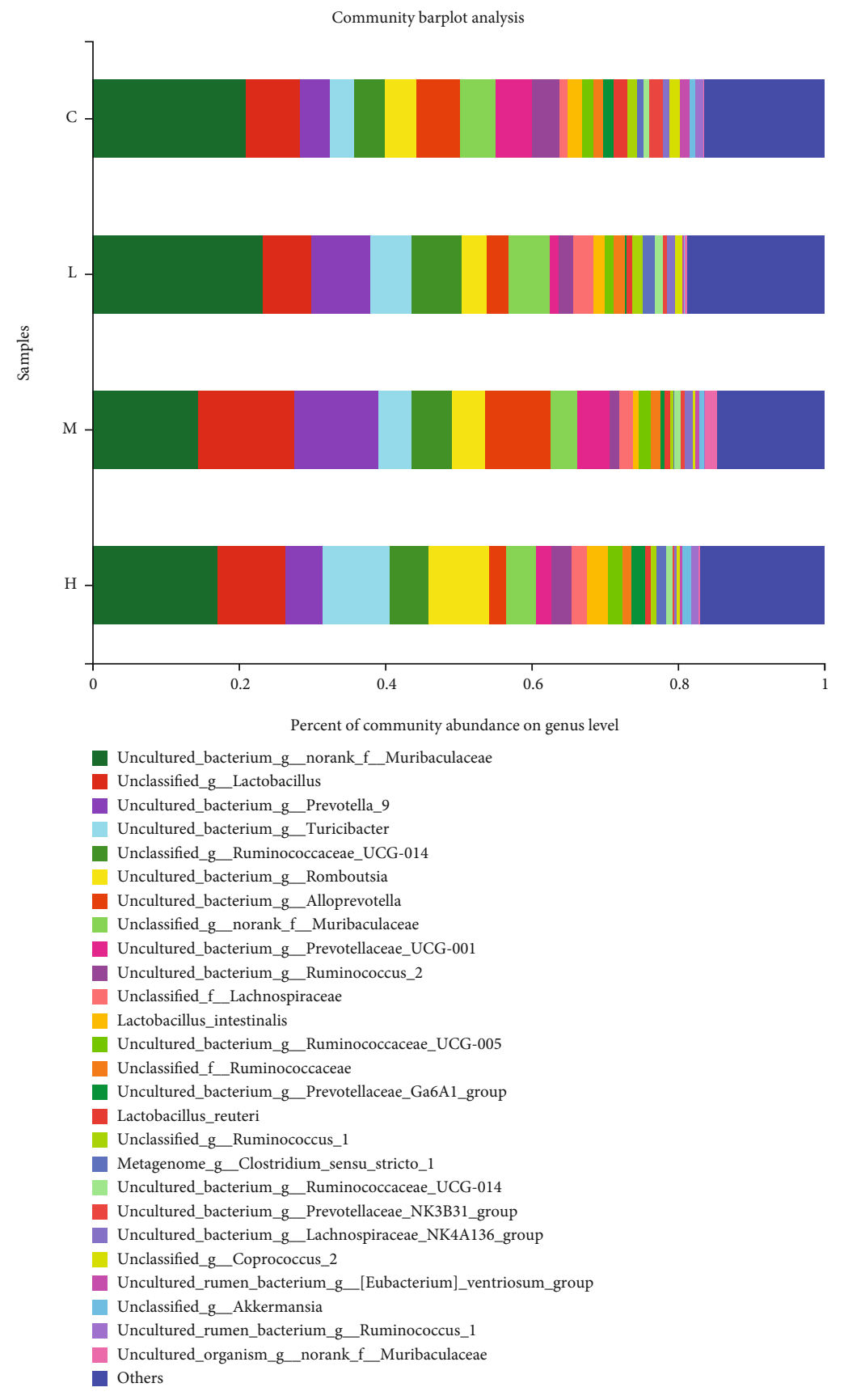

(a)

FIgURE 9: Continued. 


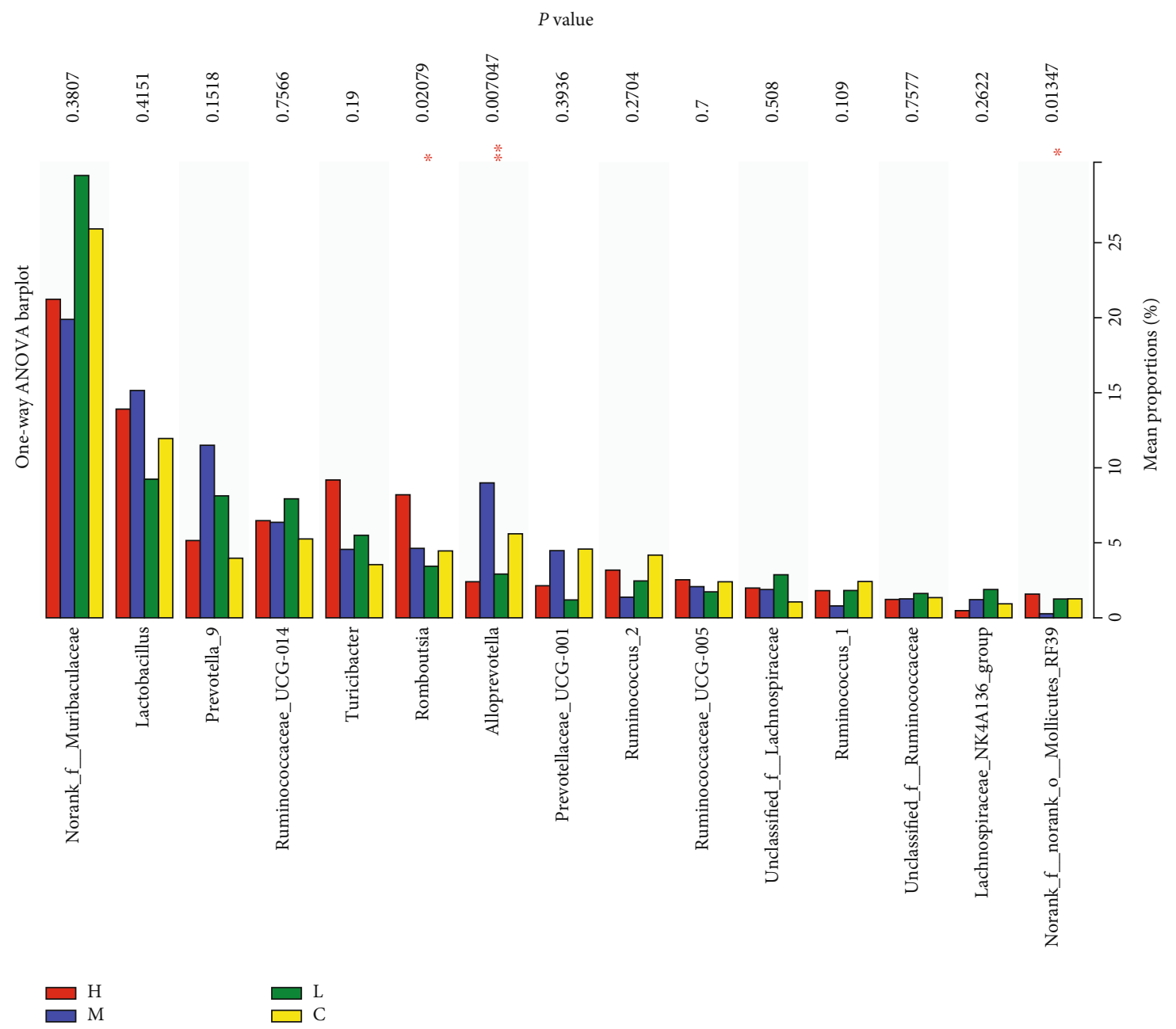

(b)
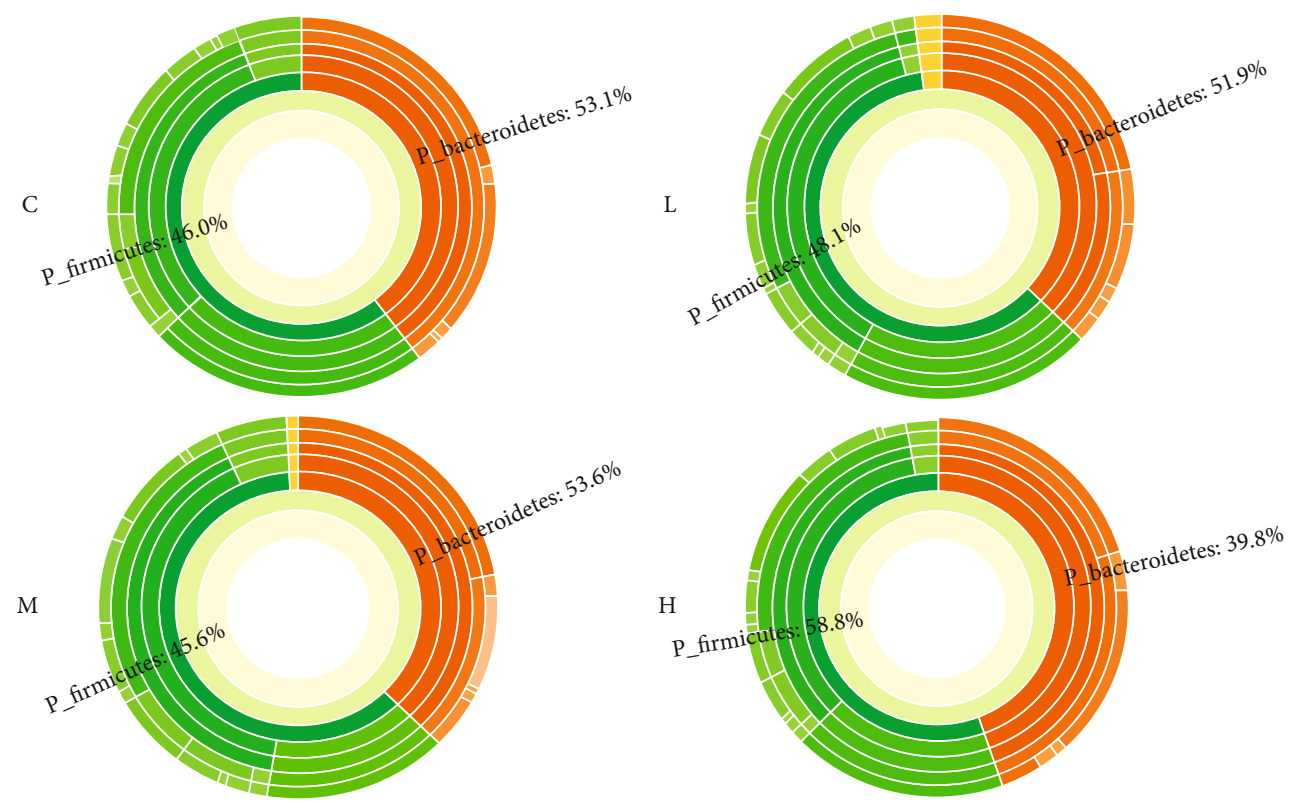

(c)

FiguRE 9: Effect of MCM on the relative abundance of gut microbiota in female rats. C: normal control group; L: water extraction of MCM $0.2 \mathrm{~g} / \mathrm{kg} / \mathrm{day}$; M: water extraction of MCM $2 \mathrm{~g} / \mathrm{kg} /$ day; $\mathrm{H}$ : water extraction of MCM $20 \mathrm{~g} / \mathrm{kg} /$ day. ${ }^{*} P<0.05$, compared with the control group; ${ }^{* *} P<0.01$, compared with the control group. 


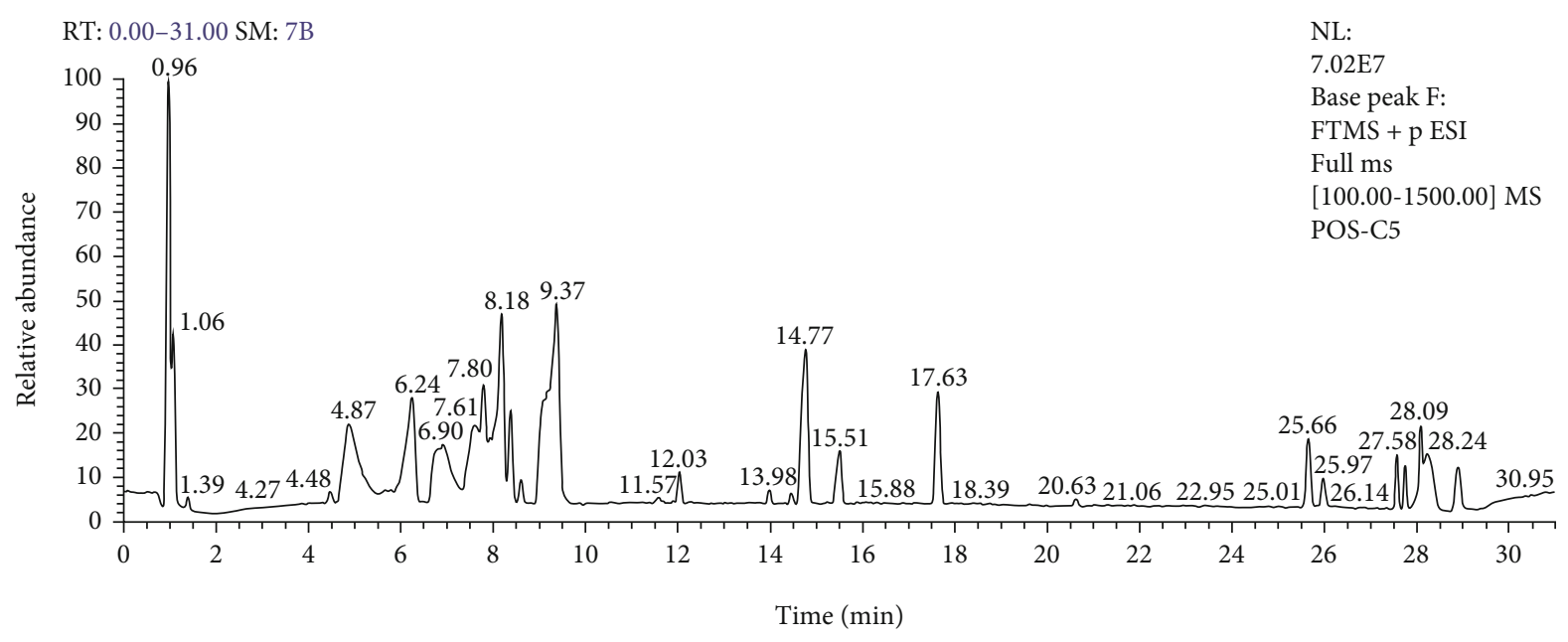

(a)

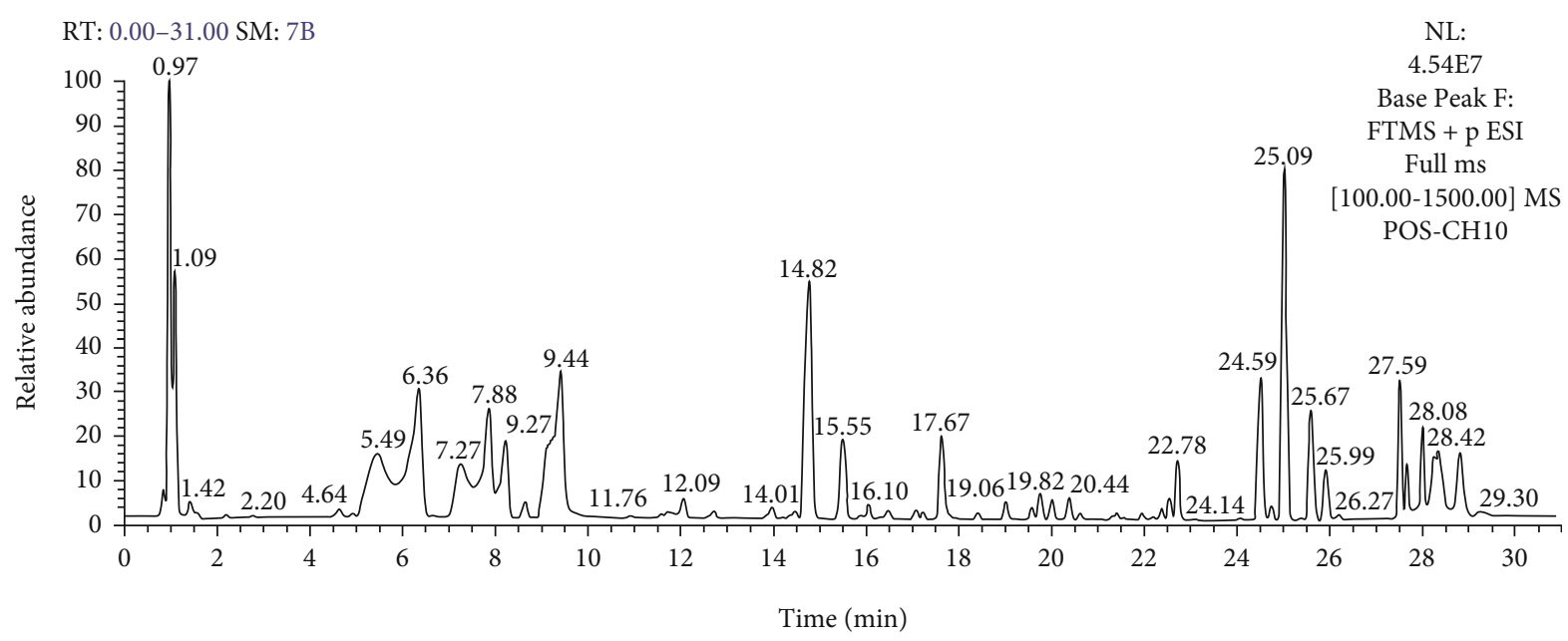

(b)

FIGURE 10: An orbitrap mass spectrometer equipped with a heated electrospray ionization (HESD) probe was coupled to the UHPLC system to intensify the chromatogram of rat urine. (a) The positive ion basic peak intensity (BPI) chromatogram of the mixed sample of the control group and the experimental group. (b) The negative ion basic peak intensity (BPI) chromatogram of the mixed sample of the control group and the experimental group.

and gastrointestinal motility index. Motilin (Mtl) is one of the hormones of the digestive system; its function is to increase the migrating motor complex (MMC), promote gastrointestinal movement, and stimulate the secretion of pepsin, while promoting gastric emptying [22, 23]. The results of this experiment showed that Mtl levels in all the treatment groups decreased, and the Mtl levels in the 2 and $20 \mathrm{~g} / \mathrm{kg} /$ day groups were significantly lower than those in the control group. Calcitonin gene-related peptide (CGRP) is a neuropeptide that is distributed in the brain and gastrointestinal system and has a variety of biological effects; it can protect gastrointestinal function by inhibiting gastric acid secretion, increasing gastric mucosal blood flow, slowing gastrointestinal movement, inhibiting inflammatory reaction, antagonizing free radical damage, and regulating gastrointestinal hormone secretion. In this experiment, it was found that the CGRP level of male rats in the $0.2,2$, and $20 \mathrm{~g} / \mathrm{kg} /$ day groups and female rats in the $20 \mathrm{~g} / \mathrm{kg} /$ day group increased, and there were significant differences with the control group. It was reported that the increase of the CGRP level could reduce the release of inflammatory factors TNF and IL-1 [24]. Therefore, the results show that MCM can inhibit the release of inflammatory cytokines and play an important role in regulating local inflammation. The results are in line with the clinical treatment of acute gastroenteritis and abdominal pain vomiting and diarrhea by MCM, which may have good resistance to intestinal accumulation heat and enhance the characteristics of intestinal peristalsis.

The levels of ALT, AST, ADA, and CHE can reflect the damage and severity of liver cells. The biochemical indicators of liver function such as TP, ALB, and CHE can reflect the anabolic function of cell protein. This indicates a decrease in ALT level in serum liver injury $[25,26]$. The levels of TB, $\mathrm{DB}$, and associates can reflect the function of liver excretion and secretion and detoxification; ALP and GGT levels can reflect cholestasis. AST and ALT are very important enzymes in the metabolic process of the body. When liver cells are 

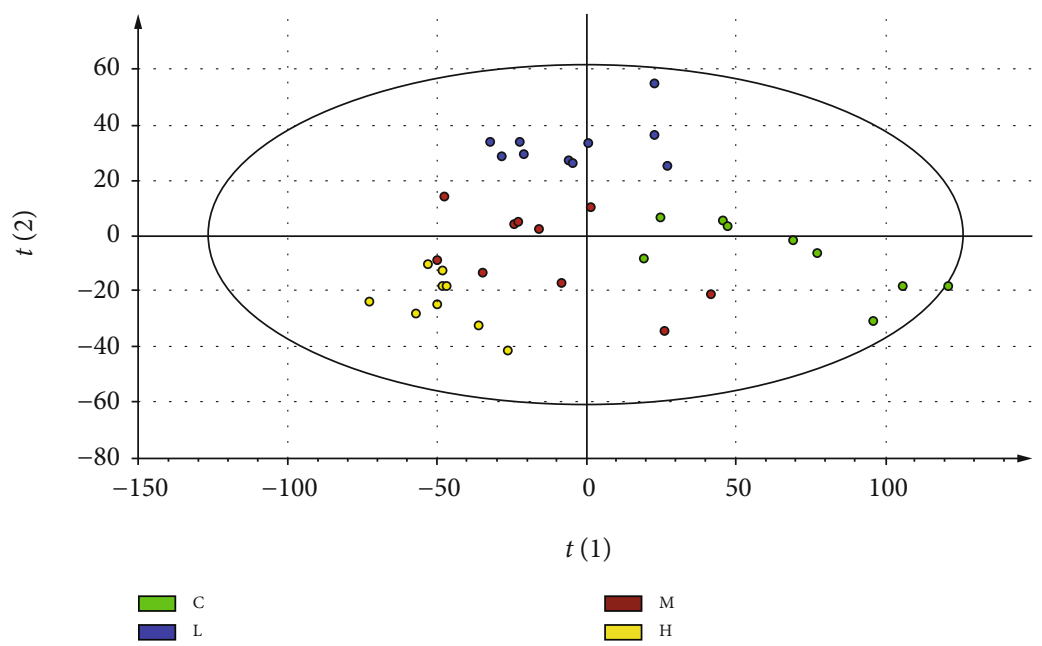

(a)
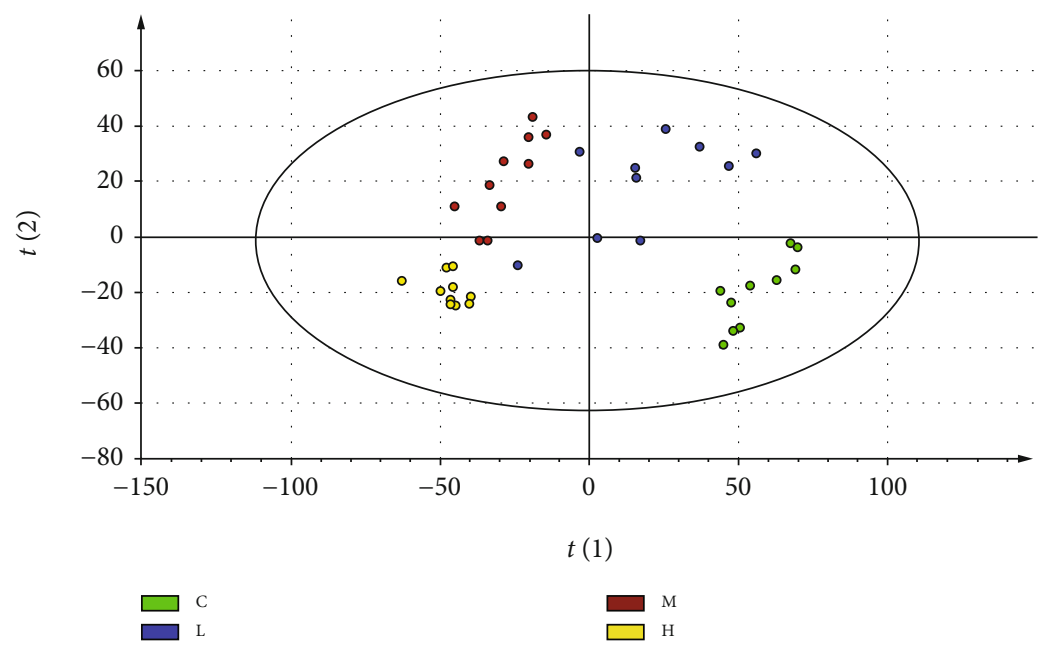

(b)

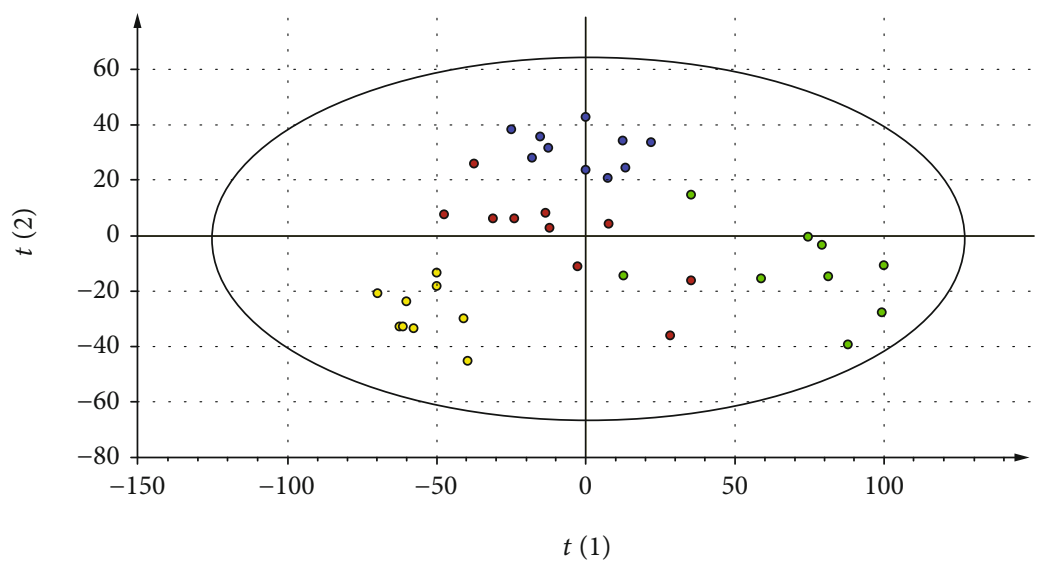

$\square \begin{aligned} & \mathrm{C} \\ & \mathrm{L}\end{aligned} \square \mathrm{M}$

(c)

FIgURE 11: Continued. 


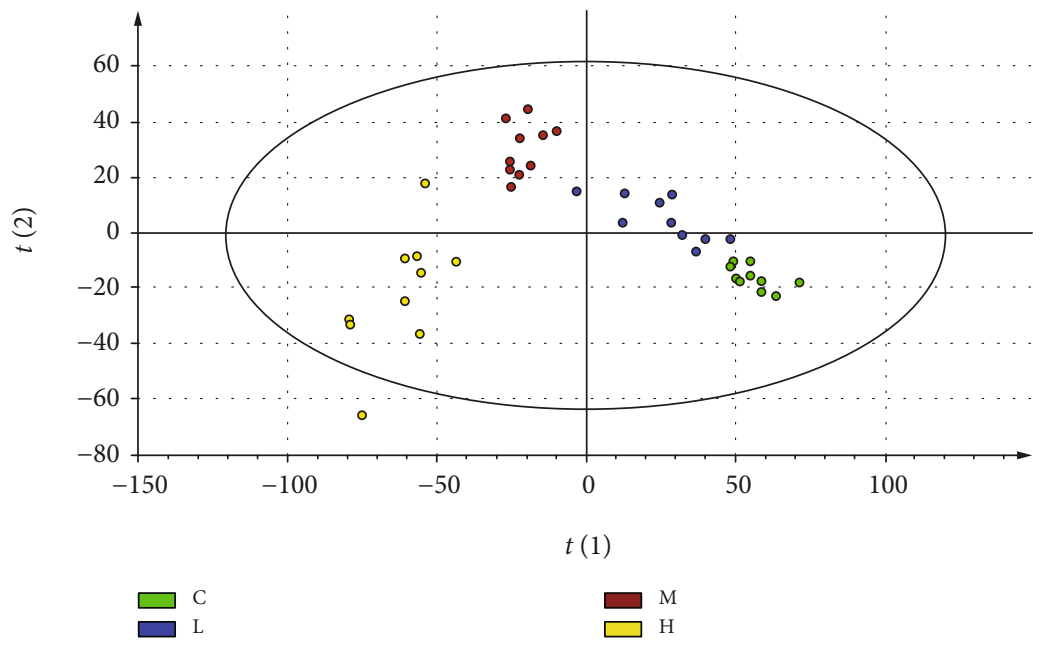

(d)

FIGURE 11: The mean PCA of the control and treated groups in positive (a, b) and in negative (c, d). (a) 12 weeks after treatment in the positive mode $(\mathrm{R} 2 \mathrm{X}[1]=0.32, \mathrm{R} 2 \mathrm{X}[2]=0.0822)$; (b) 12 weeks after treatment in the positive mode $(\mathrm{R} 2 \mathrm{X}[1]=0.241, \mathrm{R} 2 \mathrm{X}[2]=0.0919)$; (c) 12 weeks after treatment in the negative mode $(\mathrm{R} 2 \mathrm{X}[1]=0.31, \mathrm{R} 2 \mathrm{X}[2]=0.091)$; (d) 12 weeks after treatment in the negative mode $(\mathrm{R} 2 \mathrm{X}[1]=0.273$, $\mathrm{R} 2 \mathrm{X}[2]=0.0837)$.

TABLE 2: Effects of MCM on toxic biomarkers in rats.

\begin{tabular}{|c|c|c|c|c|c|c|c|c|}
\hline $\begin{array}{l}\text { Retention } \\
\text { time (min) } \\
\end{array}$ & $\begin{array}{l}\text { Measured } \\
\text { mass }(\mathrm{Da})\end{array}$ & $\begin{array}{l}\text { Calculate } \\
\text { mass (Da) }\end{array}$ & $\begin{array}{l}\text { Error } \\
(\mathrm{ppm})\end{array}$ & $\begin{array}{l}\text { Scan } \\
\text { mode }\end{array}$ & Identity & $\begin{array}{c}\text { Elemental } \\
\text { composition }\end{array}$ & $P$ value & $\begin{array}{c}\text { Fold } \\
\text { change }\end{array}$ \\
\hline 12.67 & 176.0706 & 176.0706 & 0 & + & Indoleacetic acid & $\mathrm{C} 10 \mathrm{H} 9 \mathrm{NO} 2$ & $5.8 \times 10^{-5}$ & 2.769 \\
\hline 10.26 & 208.0970 & 208.0968 & 0 & + & N-Acetyl-L-phenylalanine & $\mathrm{C} 11 \mathrm{H} 13 \mathrm{NO} 3$ & $1.9 \times 10^{-4}$ & 3.252 \\
\hline 7.55 & 162.0571 & 163.0516 & 6 & - & 2-Hydroxycinnamic acid & $\mathrm{C} 9 \mathrm{H} 8 \mathrm{O} 3$ & $3.1 \times 10^{-9}$ & 4.282 \\
\hline 5.21 & 188.0362 & 188.0353 & 4 & - & Kynurenic acid & $\mathrm{C} 10 \mathrm{H} 7 \mathrm{NO} 3$ & $1.2 \times 10^{-9}$ & 4.699 \\
\hline 3.94 & 204.0312 & 204.0302 & 4 & - & Xanthurenic acid & $\mathrm{C} 10 \mathrm{H} 7 \mathrm{NO} 4$ & $2.2 \times 10^{-8}$ & 3.355 \\
\hline
\end{tabular}

$P$ value: group of $20 \mathrm{~g} / \mathrm{kg} /$ day compared with the control group at the $90^{\text {th }}$ day. Fold change was calculated by dividing the mean of the peak intensity of each metabolite from the $20 \mathrm{~g} / \mathrm{kg} /$ day group relative to the control group at the $90^{\text {th }}$ day.

damaged, AST and ALT are released, increasing the levels of AST and ALT in the serum. However, when the increase of these two indicators does not exceed twice, there is no toxicological significance [27]. This study found that AST and ALT increased in both the female and male administration groups, and the liver organ coefficient was larger than that of the normal control group. It shows that MCM has an effect on the liver, but it is not toxic, and the pathological section shows normal.

BUN, CRE, and UA were the indicators of renal function. BUN is the end product of amino acid and protein metabolism of the body and is the main component of nonprotein in the blood. When the kidney is injured, the excretion of BUN decreases, leading to an increase in the level of BUN in the serum. CRE is a kind of small molecule product produced by muscle metabolism in the body, which can be filtered and excreted by the kidney. CRE level is an important indicator to detect the detoxification ability of the kidney [28]. In this experiment, it was found that the effects of 90 days of feeding of water extracted from MCM on renal function had no significant toxicological significance.
Being rich in the amount and type of gut microbes not only helps digestion and absorption of nutrients, it also has a huge impact on the ADME process of exogenous chemicals. At the same time, these metabolites can adversely affect the abundance and diversity of the gut microbes, and analyzing the distribution of intestinal flora is the innovation of this experiment. From the results (Figure 7), the distribution of intestinal flora in the administration group and the control group has changed. Compared with the normal control group, the flora with a significantly higher relative abundance of the MCM administration groups are Prevotella, Ruminococcaceae, and Romboutsia (Figures 8 and 9). In terms of urine metabolomics, it was found that the level of uric acid in the urinary metabolites of the administration group was significantly different from that of the normal control group; the results are similar to previous studies. Previous studies have found that intestinal flora is involved in the regulation of uric acid excretion. Short-chain fatty acids (such as butyrate) regulate the proliferation and repair of intestinal epithelial cells, change the number and distribution of uric acid transporters in the body, and affect the transport and excretion of uric acid. Lim et al. [29] conducted a horizontal study 
and analysis of fecal microbes in the general population of Korea and a two-year longitudinal study and analysis of fecal microbes in identical twin pairs and confirmed that the level of human blood uric acid and the dominant type of intestinal flora are closely related. Among them, the dominant intestinal type of Prevotella (the main decomposition products are butyric acid, succinic acid, lactic acid, etc.) has lower blood uric acid than the dominant intestinal type of Bacteroides. In 2016, Stern et al. [30] used broad-spectrum gene sequencing technology to compare and analyze the stools of 23 patients with kidney stones and 6 in the general population and also found the correlation between hemorrhagic uric acid and the abundance of Prevotella and Bacteroides. In the same year, Guo et al. [31] studied the intestinal flora and metabolites and found that the type and quantity of intestinal flora in patients with gout and the decrease in the content of broken chain fatty acid butyric acid are associated with xanthine dehydrogenase and uric acid in the intestine. The decrease in the level of uric acid in the urine may be caused by changes in the distribution of intestinal flora.

$\mathrm{N}$-Acetyl-L-phenylalanine is the product of phenylalanine $\mathrm{N}$-acetyltransferase in the phenylalanine metabolic pathway. Changes in the level of N-acetyl-L-phenylalanine indicate that exposure to MCM will affect the metabolism of phenylalanine. Indole acetic acid is the starting point and central compound of the tryptophan metabolism pathway. Tryptophan metabolism is closely related to the central nervous system and immune regulation and plays an important role in maintaining the normal physiological functions of the central nervous system. Therefore, the change of indole acetic acid content suggests that MCM can interfere with the tryptophan metabolism of normal rats. Previous studies have shown that tryptophan and its metabolites are involved in the development of liver steatosis and steatohepatitis [32]. Changes in ALT and AST levels may be related to the metabolic pathways of tryptophan.

\section{Conclusion}

Combining the above detection indicators, it is shown that the tested drug Mosla chinensis Maxim water extract is actually nontoxic and safe for clinical application. The results provide a scientific basis for the further application of MCM.

\section{Abbreviations}

MCM: Mosla chinensis Maxim

RBC: Red blood cell

WBC: White blood cell

HGB: Hemoglobin

PLT: Platelet

ALB: Albumin

TBA: Total bile acid

ALT: Alanine transaminase

TP: Total protein

ALP: Alkaline phosphatase

ADA: Adenosine deaminase

AST: Aspartate aminotransferase

CHE: Cholinesterase
TB: Total bilirubin

DB: Direct bilirubin

GGT: Glutamyl transferase

LDL: Low-density lipoprotein

TG: Triglyceride

TC: Total cholesterol

HDL: High-density lipoprotein

GLU: Glucose

BUN: Blood urea nitrogen

CRE: Creatinine

UA: Uric acid

MDA: Malondialdehyde

LPO: Lipid peroxide

GAS: Gastrin

CGRP: Calcitonin gene-related peptide

Mtl: Motilin

ET-1: Endothelin1

TNF: Tumor necrosis factor

IL-1: Interleukin 1

IgG: Immunoglobulin $\mathrm{G}$

IgA: Immunoglobulin A

IgM: Immunoglobulin $\mathrm{M}$

C4: $\quad$ Complement 4

C3: Complement 3

TCM: Traditional Chinese medicine.

\section{Data Availability}

The data used to support the findings of this study are available from the corresponding author upon request.

\section{Ethical Approval}

All experiments were carried out in adherence with the guidelines of the Institutional Animal Care and Use Committee of China and were approved by the Animal Care and Research Committee of Jiangxi University of Traditional Chinese Medicine.

\section{Consent}

The authors are accountable for all aspects of the work in ensuring that questions related to the accuracy or integrity of any part of the work are appropriately investigated and resolved. All participants agreed to publish.

\section{Conflicts of Interest}

The authors declare that they have no known competing financial interests or personal relationships that could have appeared to influence the work reported in this paper.

\section{Authors' Contributions}

Conception and design were handled by Zhiyong Liu and Shouming Li; administrative support was handled by Longxue Li and Tao Hong; provision of study materials was handled by Dan Lei, Li Liu, and Kun Shu; collection and assembly of data were handled by Shenghong Huang, Ming Zhou, and Pingdong Cai; data analysis and interpretation 
were handled by Zhouyang Gu and Dan Lei; manuscript writing was handled by all authors; and final approval of manuscript was given by all authors. Dan Lei, Longxue Li, and Shenghong Huang contributed equally to this work.

\section{Acknowledgments}

First of all, we would like to show our deepest gratitude to Hao Chen, deputy director of the pharmacy department of the Affiliated Hospital of Jiangxi University of Traditional Chinese Medicine, who helped extract the water extract of MCM. This research was supported by the Double-First Class Discipline Construction Project (No. JXSYLXK-ZHYA0138).

\section{Supplementary Materials}

Parameter of elution gradient in positive and negative ionization mode. Solvent A: $0.1 \%$ aqueous formic acid; solvent B: acetonitrile. (Supplementary Materials)

\section{References}

[1] Q. Miao, W. Fang, X. Zhang, and G. Luo, The research progress of $M$. chinensis-Jiangxiangru's chemical components and pharmacological effect, no. 2, 2015School of pharmacy,Jiangxi University of TCM, 2015.

[2] G. Mu-zi, "Comparison of pharmacological effects of volatile oil from Qin Mosla Chinensis Maxim and Mosla Chinensis Maxim," Beijing Journal of Traditional Chinese Medicine, vol. 19, no. 4, pp. 46-49, 2000.

[3] F. Stickel, E. Patsenker, and D. Schuppan, "Herbal hepatotoxicity," Journal of Hepatology, vol. 43, no. 5, pp. 901-910, 2005.

[4] W. Jingli and Z. Chaofan, "A summary of studies on Chinese herbal medicine and its preparations on liver injury," Chinese Journal of Traditional Chinese Medicine, vol. 36, no. 23, pp. 3371-3374, 2011.

[5] S. Zhang, X. Li, F. Lu, and S. Liu, "A new direction in the study of toxicity of traditional Chinese medicine: "discovery of potential toxicity of non-toxic" drugs," World Science and Technology/Modernization of Traditional Chinese Medicine and Materia Medica, vol. 3, pp. 627-632, 2015.

[6] Z. Yiliang, Proceedings of the First (2016) Academic Communication Conference of the Chinese Toxicology Committee of Chinese Medicine and Natural Medicine Toxicology, 2016.

[7] S. Port, Proceedings of the Fourth Academic Annual Meeting of the Toxicology and Safety Evaluation of Chinese Medicine and Natural Medicines by the Chinese Society of Toxicology, 2019.

[8] W. Wei, "Aristolochic acids may cause cancer, raises questions about safety of Chinese medicine," Science \& Technology Review, vol. 35, no. 21, p. 9, 2017.

[9] Y. Z. Zhang Guangping, Proceedings of the First (2016) Academic Exchange Conference of the Toxicology Committee of Chinese Medicine and Natural Medicines of the Chinese Society of Toxicology, 2016.

[10] J. Wei, Y. Chen, Y. Yu, X. Zhang, Z. Wei, and X. Ye, "Research and analysis on the safety factors of Chinese herbal compounds," Chinese Journal of Ethnomedicine and Ethnopharmacy, vol. 23, 2016.

[11] X. Xiaohe, 2019 abstracts of papers of the third academic symposium of the Professional Committee of Clinical Pharmacology and Toxicology of the Chinese Society of Integrated Chinese and Western Medicine, 2019.

[12] W. Jiabo, S. Haibo, and N. Keyong, "Challenges and reflections on safety evaluation of traditional Chinese medicine," Adverse Drug Reactions, vol. 20, pp. 409-412, 2018.

[13] Y. Gui and W. Jie, National seminar on research and reasonable application of poisonous Chinese medicine, 2012.

[14] X. Lian, N. Ai, X. Lu, and X. Fang, “Toxicogenomics and its application in safety evaluation of traditional Chinese medicine," China Journal of Chinese Materia Medica, vol. 40, no. 14, pp. 2690-2695, 2015.

[15] Z. Wang, Q. Zhang, Q. Mao, L. Liu, and S. Li, “Application of metabonomics in the evaluation of toxicity of traditional Chinese medicine," Acta Chinese Medicine and Pharmacology, vol. 42, no. 6, pp. 85-89, 2014.

[16] L. Xie, X. Fan, Z. Li et al., "Application of transcriptomic technologies in hepatotoxicity induced by Chinese materia medica," Chinese Traditional and Herbal Drugs, vol. 10, pp. 136-141, 2015.

[17] D. Tang, S. Yuan, N. Zhang, Y. Zhao, F. Wei, and Y. Zhao, "Research progress of pharmacodynamics and toxicity of traditional Chinese medicine based on urinary metabolomics," Chinese Journal of Pharmaceutical Analysis, vol. 36, no. 1, pp. 1-8, 2016.

[18] N. Qi, Y. Liu, C. He, J. Hua, and J. Li, "Metabonomic study on the toxicity of Chinese medicine (Il) hepato nephrotoxicity of Pyrrolizidine alkaloid," Journal of Nanjing University of Traditional Chinese Medicine, vol. 5, pp. 54-57, 2012.

[19] T. Hong, L. X. Li, X. P. Han et al., "Effect of Astragalus membranaceus oral solution on lifespan and learning and memory ability of honey bees," BioMed Research International, vol. 2020, Article ID 5745048, 7 pages, 2020.

[20] Z. Jiang, W. Xu, W. Jin et al., "Normal ranges for hematology, biochemical indexes and organ-body ratios of SD rats," Journal of Preventive Medicine Information, vol. 10, pp. 45-47, 2011.

[21] Z. Lijuan, Yunnan wild Elsholtzia oil regulates blood lipid and its effects on the learning and memory of offspring mice, Kunming Medical College, 2011.

[22] T. Haba and S. K. Sarna, "Regulation of gastroduodenal emptying of solids by gastropyloroduodenal contractions," American Journal of Physiology-Gastrointestinal and Liver Physiology, vol. 264, no. 2, pp. G261-G271, 1993.

[23] S. Melmed, K. S. Polonsky, P. R. Larsen, and H. M. Kronenberg, Williams Textbook of Endocrinology, Elsevier Health Sciences, 2015.

[24] M.-F. Xiao, Z. Lin, L. Zhe, and Q. Liu, "The role of calcitoninrelated gene peptide in the regulation of mouse macrophage inflammation," Chinese Journal of Modern Medicine, vol. 29, no. 13, pp. 18-24, 2019.

[25] Y. Zhang, X. Lin, Y. Wang, W. La, and X. Chen, "Column chromatographyanalysison the protective effect of albuminon liver-injured in rats," Chinese Journal of Blood Transfusion, vol. 31, no. 10, pp. 46-50, 2018.

[26] L. Xian-ping, Y. Wei-yan, and Z. Cai-yan, "What's new in the application of human serum albumin in the treatment of patients with end stage liver diseases," Journal of Practical Hepatology, vol. 20, no. 1, pp. 115-119, 2017.

[27] M. Yimin, L. Xiaolin, and C. Chengwei, "Introduction to the 2013 U.S. FDA guidelines for drug-induced liver injury-premarket clinical evaluation of pharmaceutical R\&D companies," Liver, vol. 5, no. 2013, pp. 325-330, 2013. 
[28] Y. Zhao, L. Yang, L. Zhang, and P. Fu, “A combined biomarker of urinary neutrophil gelatinase-associated lipocalin and serum creatinine for the prediction of acute kidney injury: what else can we know?," Journal of Critical Care, vol. 54, pp. 280-281, 2019.

[29] M. Y. Lim, M. Rho, Y. M. Song, K. Lee, J. Sung, and G. P. Ko, "Stability of gut enterotypes in Korean monozygotic twins and their association with biomarkers and diet," Scientific Reports, vol. 4, no. 1, article 7348, 2015.

[30] J. M. Stern, S. Moazami, Y. Qiu et al., "Evidence for a distinct gut microbiome in kidney stone formers compared to nonstone formers," Urolithiasis, vol. 44, no. 5, pp. 399-407, 2016.

[31] Z. Guo, J. Zhang, Z. Wang et al., "Intestinal microbiota distinguish gout patients from healthy humans," Scientific Reports, vol. 6 , no. 1, article 20602, 2016.

[32] Y. Ritze, G. Bárdos, A. Hubert, M. Böhle, and S. C. Bischoff, "Effect of tryptophan supplementation on diet-induced nonalcoholic fatty liver disease in mice," British Journal of Nutrition, vol. 112, no. 1, pp. 1-7, 2014. 